\title{
Robinson instabilities with a higher-harmonic cavity
}

\author{
R. A. Bosch, K. J. Kleman, and J. J. Bisognano \\ Synchrotron Radiation Center, University of Wisconsin-Madison, 3731 Schneider Drive, Stoughton, Wisconsin 53589
}

(Received 10 January 2001; published 10 July 2001)

\begin{abstract}
A radio frequency system with a fourth-harmonic "Landau" cavity suppresses coupled-bunch instabilities and increases the beam lifetime of the Aladdin electron storage ring. When the storage ring is operated with a small momentum compaction, instabilities limit the utility of the Landau cavity. Analytical modeling of instability frequencies and growth rates, simulations, and experiments suggest that the observed instabilities result from coupling between dipole and quadrupole Robinson modes.
\end{abstract}

DOI: 10.1103/PhysRevSTAB.4.074401

PACS numbers: 29.27.Bd, 29.20.Dh, 41.75.Ht

\section{INTRODUCTION}

A radio frequency cavity with resonant frequency near a harmonic of the fundamental rf cavity may increase Landau damping of synchrotron oscillations and lengthen the bunch, thereby suppressing coupled-bunch instabilities and increasing the Touschek lifetime [1-9]. A Landau cavity may be operated in passive mode, where its voltage is induced by the beam current, or active mode, where its voltage is maintained by an rf power supply and feedback. In either case, quiet operation requires avoidance of Robinson instabilities [3,5,10-16], which are coupled-bunch instabilities where all bunches oscillate in unison.

Since 1995, a fourth-harmonic cavity has been operated at the Aladdin $800 \mathrm{MeV}, 300 \mathrm{~mA}$ electron storage ring, suppressing coupled-bunch instabilities and doubling the beam lifetime [6]. Passive-mode operation is stable with beam currents exceeding $120 \mathrm{~mA}$. Experimentally, tuning in the Landau cavity at lower beam currents results in instability before optimal bunch lengthening is obtained, in approximate agreement with the predicted onset of the dipole Robinson instability [5,17]. Active operation successfully provides optimal bunch lengthening at all values of the beam current $(0-300 \mathrm{~mA})$ when using feedback on the fundamental and Landau cavities that is predicted to avoid dipole Robinson instabilities [11].

To increase the brightness of Aladdin's synchrotron radiation, a low-emittance mode of operation is being developed. Successful operation of the Landau cavity is required to suppress coupled-bunch and microwave instabilities and to obtain a lifetime comparable to the standard Aladdin lattice. In low-emittance mode with the "LF20" lattice, the momentum compaction of 0.0043 is about $1 / 8$ of its value of 0.0335 in the standard Aladdin lattice, reducing the synchrotron frequency by a factor of $\sim 3$ [18]. Experimentally, passive operation of the Landau cavity is unstable with optimally lengthened bunches, but stable at ring currents of $80-300 \mathrm{~mA}$ when the bunches are lengthened beyond optimal to assume a double-hump shape. Active operation with optimally lengthened bunches is unstable at ring currents exceeding $\sim 100 \mathrm{~mA}$; instabilities include an oscillation of the bunch position and length.
By modeling the coupling between dipole and quadrupole Robinson modes, the observed instabilities are predicted. Simulations and experiments show instabilities in which the bunch centroid and length oscillate with comparable magnitudes. Experimental data show that instability results when the oscillation frequencies of two collective modes converge, consistent with a coupled dipole-quadrupole instability.

In Sec. II, we discuss formulas for the bunch length and Robinson instabilities. In Secs. III and IV, passive operation of the Landau cavity is studied using analytic modeling, simulations, and experiment. Sections V and VI describe active operation of the Landau cavity, while Sec. VII discusses our results.

\section{ROBINSON INSTABILITY FORMULAS}

\section{A. Synchrotron frequency and bunch length}

Consider a bunch confined in a double-rf system consisting of a fundamental cavity and a higher-harmonic cavity. Let Cavity 1 be the fundamental rf cavity with resonant frequency $\omega_{1}$ near $\omega_{g}$, the rf generator frequency. Let $Q_{1}$ be its quality factor, $R_{1}$ its impedance at resonance ( $1 / 2$ of the "accelerator" definition of shunt impedance), and $\phi_{1}$ its tuning angle $\left(-90^{\circ}<\phi_{1}<90^{\circ}\right)$, defined by $\tan \phi_{1}=2 Q_{1}\left(\omega_{g}-\omega_{1}\right) / \omega_{1}$. This tuning angle is the same as that used by Marchand [14] and Sands [19], and the negative of that used by Wilson [20]. The cavity impedance at frequency $\omega_{g}$ is $R_{1} \cos \phi_{1} e^{i \phi_{1}} . R_{1}$ and $Q_{1}$, which describe the loaded cavity including fast rf feedback, equal $1 /\left(1+\beta_{1}\right)$ times the unloaded values $R_{1}^{0}$ and $Q_{1}^{\mathrm{o}}$, where $\beta_{1}$ is the equivalent $\mathrm{rf}$ coupling coefficient of Cavity $1[17,19,20]$.

Cavity 2 is a higher harmonic cavity with resonant frequency $\omega_{2}$ near $\nu \omega_{g}$, where $\nu$ is its harmonic number. The unloaded impedance and quality factor are $R_{2}^{\mathrm{o}}$ and $Q_{2}^{\mathrm{o}}$, while the equivalent $\mathrm{rf}$ coupling coefficient of Cavity 2 is $\beta_{2}$. $Q_{2}$ is the loaded quality factor equaling $Q_{2}^{\mathrm{o}} /\left(1+\beta_{2}\right), R_{2}$ is the loaded impedance at resonance given by $R_{2}^{0} /\left(1+\beta_{2}\right)$, and $\phi_{2}$ is the tuning angle, which obeys $\tan \phi_{2}=2 Q_{2}\left(\nu \omega_{g}-\omega_{2}\right) / \omega_{2}$. 
We let $e>0$ represent the magnitude of the electronic charge, $T_{\mathrm{o}}$ the storage ring recirculation time, $\omega_{\mathrm{o}}=2 \pi / T_{\mathrm{o}}$ the angular revolution frequency, $E$ the storage ring energy, $\sigma_{E}$ the natural electron energy spread, $\alpha$ the momentum compaction, and $I>0$ the ring current. For symmetric bunches, $F_{1}$ and $F_{2}$ denote the bunch form factors in Cavities 1 and 2 for bunch length $\sigma_{t}[5,20]$. We let $V_{T 1}$ and $V_{T 2}$ denote the peak voltages in Cavities 1 and 2 , while $\psi_{1}$ and $\psi_{2}$ denote the synchronous phases in each cavity, equaling zero for a bunch passing through the cavity at the peak accelerating voltage. This definition of $\psi_{1}$ and $\psi_{2}$ is the same as that used by Sands [19] and Wilson [20], and it is the complement of the phase angle used by some authors. A synchronous electron receives an energy of $e\left(V_{T 1} \cos \psi_{1}+V_{T 2} \cos \psi_{2}\right)=e V_{s}$ when passing through the rf cavities, and radiates (on average) an energy of $e V_{s}$ per revolution, where $V_{s}$ is the synchronous voltage.

Choosing a time coordinate where $t=0$ is a synchronous time, the accelerating voltage experienced by an electron passing through the rf cavities at time $t$ is

$$
V(t)=V_{T 1} \cos \left(\psi_{1}+\omega_{g} t\right)+V_{T 2} \cos \left(\psi_{2}+\nu \omega_{g} t\right),
$$

where $V(0)=V_{s}$. Neglecting radiation damping and the random nature of synchrotron radiation emission, the arrival time of a single electron $(\tau)$ relative to the synchronous time obeys

$$
\frac{d^{2} \tau}{d t^{2}}=\frac{\alpha e}{E T_{\mathrm{o}}}\left[V(\tau)-V_{s}\right]=-\frac{d U}{d \tau}
$$

where

$$
U(\tau)=-\frac{\alpha e}{E T_{\mathrm{o}}} \int_{0}^{\tau}\left[V\left(t^{\prime}\right)-V_{s}\right] d t^{\prime}
$$

is the synchrotron effective potential. The potential may be expanded in a Taylor series $U(\tau)=a \tau^{2}+b \tau^{3}+c \tau^{4}+$ $\cdots$, where [5]

$$
\begin{gathered}
a=\frac{\alpha e \omega_{g}}{2 E T_{\mathrm{o}}}\left(V_{T 1} \sin \psi_{1}+\nu V_{T 2} \sin \psi_{2}\right), \\
b=\frac{\alpha e \omega_{g}^{2}}{6 E T_{\mathrm{o}}}\left(V_{T 1} \cos \psi_{1}+\nu^{2} V_{T 2} \cos \psi_{2}\right), \\
c=-\frac{\alpha e \omega_{g}^{3}}{24 E T_{\mathrm{o}}}\left(V_{T 1} \sin \psi_{1}+\nu^{3} V_{T 2} \sin \psi_{2}\right) .
\end{gathered}
$$

When $a \sigma_{t}^{2} \gg\left|b \sigma_{t}^{3}\right|$ and $\left|c \sigma_{t}^{4}\right|$, the synchrotron potential is "mostly quadratic" in the region occupied by the bunch [5].

For small oscillations, the linear synchrotron frequency $\omega_{s} \geq 0$ therefore obeys

$$
\omega_{s}^{2}=2 a=\frac{\alpha e \omega_{g}}{E T_{\mathrm{o}}}\left(V_{T 1} \sin \psi_{1}+\nu V_{T 2} \sin \psi_{2}\right) .
$$

Defining $\xi=-\nu V_{T 2} \sin \psi_{2} / V_{T 1} \sin \psi_{1}$ as the negative of the ratio of the Landau-cavity longitudinal "force" to that from the fundamental cavity [13], Eq. (7) may be written as

$$
\omega_{s}^{2}=\frac{\alpha e \omega_{g}}{E T_{\mathrm{o}}}(1-\xi) V_{T 1} \sin \psi_{1} .
$$

For $0<\xi<1$, the synchrotron frequency is reduced and the bunch is lengthened by the harmonic cavity. For $\xi=1$, the forces from the two cavities cancel; there is no net linear restoring force and the linear synchrotron frequency is zero. A long bunch length confined in a cubic + quartic potential may thereby be obtained; we refer to the case $\xi=1$ as "optimal bunch lengthening." When $\xi>1$, the synchrotron potential is a double well with two minima, resulting in a double-hump bunch shape.

For bunch lengthening, the $\mathrm{rf}$ phases obey $0^{\circ}<\psi_{1}<$ $90^{\circ}$ and $-180^{\circ}<\psi_{2}<-90^{\circ}$ for either passive operation of the higher-harmonic cavity [5] or for active operation in which the cubic term $(b)$ of the synchrotron potential is made to equal zero [11]. When the fundamental cavity is operated in the "compensated condition" where the rf current is in phase with the cavity voltage $[19,20]$, its tuning angle obeys $0^{\circ}<\phi_{1}<90^{\circ}$ (i.e., $\omega_{1}<\omega_{g}$ ). In contrast, passive operation of the harmonic cavity or active operation in the compensated condition yields $-90^{\circ}<\phi_{2}<0^{\circ}$ (i.e., $\nu \omega_{g}<\omega_{2}$ ) for bunch lengthening.

The synchrotron potential is occupied to the "filling height" $U_{\mathrm{o}}=\alpha^{2}\left(\sigma_{E} / E\right)^{2} / 2$, and the bunch length obeys $\sigma_{t}^{2}=\left\langle\tau^{2}\right\rangle-\langle\tau\rangle^{2} \approx\left\langle\tau^{2}\right\rangle$, where [1,15,21]

$$
\left\langle\tau^{n}\right\rangle=\frac{\int \tau^{n} \exp \left[-U(\tau) / 2 U_{\mathrm{o}}\right] d \tau}{\int \exp \left[-U(\tau) / 2 U_{\mathrm{o}}\right] d \tau} .
$$

In Eq. (8), the integration should be taken over an interval in $\tau$ that greatly exceeds $\sigma_{t}$, but is smaller than the confining rf bucket. Equation (8) gives, for a quadratic synchrotron potential $\left[U(\tau)=a \tau^{2}\right], \sigma_{t}=\alpha\left(\sigma_{E} / E\right) / \omega_{s}$, and, for a quartic synchrotron potential $\left[U(\tau)=c \tau^{4}\right]$, $\sigma_{t}=0.69\left(U_{\mathrm{o}} / c\right)^{1 / 4}$.

\section{B. Collective oscillations}

In a quadratic synchrotron potential, where the frequency of a synchrotron oscillation is independent of amplitude, the natural frequency of a collective bunch oscillation is the linear synchrotron frequency. In a general synchrotron potential produced by two rf cavities, we estimate the collective dipole oscillation frequency (the "Robinson" frequency) by approximating the dipole motion as a small rigid oscillation of a symmetric bunch. Neglecting the rf voltage induced by the motion (the excited rf sidebands) yields 


$$
\begin{aligned}
\frac{d^{2}\langle\tau\rangle}{d t^{2}} & =\frac{\alpha e}{E T_{\mathrm{o}}}\left[\left\langle V(\tau)-V_{s}\right\rangle\right]=\frac{\alpha e}{E T_{\mathrm{o}}}\left[V_{T 1}\left\langle\cos \left(\psi_{1}+\omega_{g} \tau\right)\right\rangle+V_{T 2}\left\langle\cos \left(\psi_{2}+\nu \omega_{g} \tau\right)\right\rangle-V_{s}\right] \\
& =\frac{\alpha e}{E T_{\mathrm{o}}}\left[F_{1} V_{T 1} \cos \left(\psi_{1}+\omega_{g}\langle\tau\rangle\right)+F_{2} V_{T 2} \cos \left(\psi_{2}+\nu \omega_{g}\langle\tau\rangle\right)-V_{s}\right] \\
& =\frac{-\alpha e \omega_{g}}{E T_{\mathrm{o}}}\left(F_{1} V_{T 1} \sin \psi_{1}+\nu F_{2} V_{T 2} \sin \psi_{2}\right)\langle\tau\rangle,
\end{aligned}
$$

where $\langle\ldots\rangle$ denotes the average over all electrons in a symmetric bunch. Thus, the frequency of collective dipole oscillations $\omega_{R}$ approximately obeys

$$
\begin{aligned}
\omega_{R}^{2} & =\frac{\alpha e \omega_{g}}{E T_{\mathrm{o}}}\left(F_{1} V_{T 1} \sin \psi_{1}+\nu F_{2} V_{T 2} \sin \psi_{2}\right) \\
& =\frac{\alpha e \omega_{g}}{E T_{\mathrm{o}}}\left(F_{1}-\xi F_{2}\right) V_{T 1} \sin \psi_{1}
\end{aligned}
$$

For a quadratic synchrotron potential (in which $F_{1}=$ $\left.F_{2}=1\right), \omega_{R}=\omega_{s}$.

A restoring force for collective dipole oscillations is predicted when $\omega_{R}^{2}>0$, which occurs for values of $\xi<$ $F_{1} / F_{2}$, where $F_{1} / F_{2}$ exceeds 1 because of the smaller form factor in the Landau cavity. Phase stability may therefore be obtained for small beam currents when the bunch is optimally lengthened with $\xi=1$, or when a double-hump bunch is obtained with $1<\xi<F_{1} / F_{2}$.

For $\xi=1$, approximating the form factors in Eq. (10) as $F_{1}=1-\left(\omega_{g} \sigma_{t}\right)^{2} / 2, F_{2}=1-\left(\nu \omega_{g} \sigma_{t}\right)^{2} / 2$ gives

$$
\omega_{R}^{2} \approx \frac{\alpha e \omega_{g}^{3}}{2 E T_{\mathrm{o}}}\left(\nu^{2}-1\right) \sigma_{t}^{2} V_{T 1} \sin \psi_{1}
$$

Equation (11) yields a dipole Robinson frequency $10 \%$ higher than that obtained by the Vlasov equation for a quartic potential, where rigidity of the bunch oscillation is not assumed (see Appendix A).

Without a harmonic cavity $(\xi=0)$, Eqs. (8) and (10) indicate that $\sigma_{t}$ and $\omega_{R}$ are proportional to $\alpha^{1 / 2}$. The lowemittance LF20 lattice ( $\alpha=0.0043)$ is therefore expected to have bunch length and Robinson frequency equaling $36 \%$ of the values obtained with the standard Aladdin lattice $(\alpha=0.0335)$ at the same rf voltage. With a harmonic cavity utilized for optimal bunch lengthening $(\xi=1)$, Eqs. (8) and (11) indicate that $\sigma_{t} \propto \alpha^{1 / 4}$ while $\omega_{R} \propto$ $\alpha^{3 / 4}$. For the case of optimal bunch lengthening, the expected LF20 bunch length is $60 \%$ of that obtained with the standard lattice, while the Robinson frequency is only $21 \%$ of that obtained with the standard lattice at the same rf voltage. The large decrease in the Robinson frequency may increase the susceptibility to Robinson instabilities when the low-emittance lattice is utilized.

\section{Single-mode Robinson instability formulas}

To approximately model Robinson instabilities in a general synchrotron potential produced by two rf cavities, we substitute the rigid dipole estimate of the collective oscillation frequency $\left(\omega_{R}\right)$ in place of the linear synchrotron frequency $\left(\omega_{s}\right)$ in a formula obtained for a quadratic potential [22]. In this model, the real angular frequency $\Omega$ and damping rate $\alpha_{R}$ of the $\mu_{\mathrm{o}}$ th Robinson mode approximately obey, for $\left|\alpha_{R}\right| \ll|\Omega|$,

$$
\left(\Omega-i \alpha_{R}\right)^{2}=\left(\mu_{\mathrm{o}} \omega_{R}\right)^{2}+\frac{i \alpha e \omega_{\mathrm{o}}^{2} I}{\pi E} \frac{\mu_{\mathrm{o}}\left(\omega_{\mathrm{o}} \sigma_{t}\right)^{2 \mu_{\mathrm{o}}-2}}{2^{\mu_{\mathrm{o}}}\left(\mu_{\mathrm{o}}-1\right) !} \sum_{n} n^{2 \mu_{\mathrm{o}}-1} F_{n \omega_{\mathrm{o}}}^{2} Z\left(n \omega_{\mathrm{o}}+\Omega\right),
$$

where $Z$ is the ring impedance, $F_{n \omega_{\mathrm{o}}}$ is the bunch form factor at angular frequency $n \omega_{\mathrm{o}}$, and the sum is over $n=M j$ $(j=-\infty, \ldots, \infty)$, where $M=\omega_{g} / \omega_{\mathrm{o}}$ is the number of $\mathrm{rf}$ buckets. The modes with $\mu_{\mathrm{o}}=1,2,3$, and 4 are the dipole, quadrupole, sextupole, and octupole modes, respectively.

For the case of two rf cavities, we keep the dominant terms in the sum over $Z\left(n \omega_{0}+\Omega\right)$, where $n= \pm M, \pm \nu M$, to obtain a formula for the $\mu_{\mathrm{o}}$ th mode frequency

$$
\Omega^{2}=\left(\mu_{\mathrm{o}} \omega_{R}\right)^{2}-\frac{\alpha e \omega_{g} I}{E T_{\mathrm{o}}} \frac{\mu_{\mathrm{o}}\left(\omega_{g} \sigma_{t}\right)^{2 \mu_{\mathrm{o}}-2}}{2^{\mu_{\mathrm{o}}}\left(\mu_{\mathrm{o}}-1\right) !}\left[R_{1} F_{1}^{2}\left(\sin 2 \phi_{1-}+\sin 2 \phi_{1+}\right)+\nu^{2 \mu_{\mathrm{o}}-1} R_{2} F_{2}^{2}\left(\sin 2 \phi_{2-}+\sin 2 \phi_{2+}\right)\right] .
$$

The sideband tuning angles are defined by $\tan \phi_{1 \pm}=2 Q_{1}\left(\omega_{g} \pm \Omega-\omega_{1}\right) / \omega_{1}$ and $\tan \phi_{2 \pm}=2 Q_{2}\left(\nu \omega_{g} \pm \Omega-\right.$ $\left.\omega_{2}\right) / \omega_{2}$. A zero-frequency instability onsets when $\Omega^{2} \rightarrow 0$, in which case $\phi_{1 \pm} \rightarrow \phi_{1}$ and $\phi_{2 \pm} \rightarrow \phi_{2}$. Zero-frequency instability is therefore predicted when

$$
\omega_{R}^{2}<\frac{\alpha e \omega_{g} I}{E T_{\mathrm{o}}} \frac{\left(\omega_{g} \sigma_{t}\right)^{2 \mu_{\mathrm{o}}-2}}{2^{\mu_{\mathrm{o}}-1} \mu_{\mathrm{o}} !}\left(R_{1} F_{1}^{2} \sin 2 \phi_{1}+\nu^{2 \mu_{\mathrm{o}}-1} R_{2} F_{2}^{2} \sin 2 \phi_{2}\right) .
$$


The dipole mode zero-frequency instability criterion may also be obtained as the condition under which the rf generator voltages do not provide a restoring force for slow rigid bunch motions in which the wake fields move with the bunch [19]; the instability is known accordingly as the equilibrium phase instability [3].

When zero-frequency instability is avoided, the Robinson damping rate is given by

$$
\alpha_{R}=\frac{\alpha e \omega_{g} I}{\Omega E T_{\mathrm{o}}} \frac{\mu_{\mathrm{o}}\left(\omega_{g} \sigma_{t}\right)^{2 \mu_{\mathrm{o}}-2}}{2^{\mu_{\mathrm{o}}}\left(\mu_{\mathrm{o}}-1\right) !}\left[F_{1}^{2} R_{1}\left(\cos ^{2} \phi_{1-}-\cos ^{2} \phi_{1+}\right)+\nu^{2 \mu_{\mathrm{o}}-1} F_{2}^{2} R_{2}\left(\cos ^{2} \phi_{2-}-\cos ^{2} \phi_{2+}\right)\right] .
$$

Using the definitions of $\phi_{1 \pm}$ and $\phi_{2 \pm}$, Eq. (15) may be written in the form

$$
\alpha_{R}=\frac{8 \alpha e I}{E T_{\mathrm{o}}} \frac{\mu_{\mathrm{o}}\left(\omega_{g} \sigma_{t}\right)^{2 \mu_{\mathrm{o}}-2}}{2^{\mu_{\mathrm{o}}}\left(\mu_{\mathrm{o}}-1\right) !}\left(F_{1}^{2} R_{1} Q_{1} \tan \phi_{1} \cos ^{2} \phi_{1+} \cos ^{2} \phi_{1-}+\nu^{2 \mu_{\mathrm{o}}-2} F_{2}^{2} R_{2} Q_{2} \tan \phi_{2} \cos ^{2} \phi_{2+} \cos ^{2} \phi_{2-}\right),
$$

showing that $\alpha_{R}$ approaches a finite limit as $\Omega^{2} \rightarrow 0$. A negative value of $\alpha_{R}$ indicates growth. Because $0^{\circ}<\phi_{1}<90^{\circ}$ and $-90^{\circ}<\phi_{2}<0^{\circ}$ for bunch lengthening, the fundamental cavity contributes to Robinson damping while the higher-harmonic cavity contributes to Robinson growth. Radiation damping increases the damping rate of the $\mu_{\mathrm{o}}$ th mode by $\mu_{\mathrm{o}} \tau_{L}^{-1}$, where $\tau_{L}$ is the longitudinal radiation damping time which obeys $\tau_{L}^{-1} \ll|\Omega|$.

Equations (13)-(16) may be obtained directly for the dipole mode by assuming a rigid bunch motion in the synchrotron potential produced by two rf cavities [5].

For the dipole mode of an optimally lengthened bunch, Sec. II B indicates that $\omega_{R}^{2} \propto \alpha^{3 / 2}$, while the second term on the right-hand side (RHS) of Eq. (13) is approximately $\propto \alpha$. Consequently, real frequency shifts comparable to $\omega_{R}$ may result for sufficiently small $\alpha$. This may cause the equilibrium phase instability (when $\Omega \rightarrow 0$ ) or strong coupling to the quadrupole mode (when $\left.\Omega \rightarrow 2 \omega_{R}\right)$.

Because the above Robinson-mode results are valid for a quadratic synchrotron potential (where $\omega_{R}=\omega_{s}$ ), approximate validity is expected for low Landau-cavity voltages with $\xi \ll 1$, in which case the potential is mostly quadratic [consisting of a quadratic confining potential with relatively small cubic and quartic terms given by Eqs. (5) and (6)]. For a quartic synchrotron potential with $\xi=1$, the above results are in approximate agreement with Vlasov-equation results (see Appendix A) for the dipole and quadrupole modes. The above sextupole and octupole results overestimate the frequency shifts (real and imaginary) in a quartic potential, and therefore may overestimate instability growth rates.

The approximate validity of the above formulas for dipole and quadrupole Robinson modes in both quartic and quadratic synchrotron potentials suggests that Eqs. (12)-(16) will provide a good approximation for these modes in a general synchrotron potential with $\xi \leq 1$. For $\xi>1$, a double-hump bunch is formed in which a given electron may be confined to a single hump. Because our model does not account for any associated modification of the dynamics, its accuracy may be reduced for double-hump bunches with $\xi>1$.

\section{Coupling between the dipole and quadrupole Robinson modes}

In modeling a passive harmonic cavity, we find that the quadrupole Robinson frequency may be comparable to the dipole Robinson frequency, so that coupling of these modes may occur [12,22]. To better model these modes, dipole-quadrupole mode coupling may be included in their frequencies and growth rates by using Eqs. (B11)-(B13) of Appendix B. When the mode coupling is insignificant, these formulas reproduce the single-mode dipole and quadrupole results of Sec. IIC. We refer to the coupled modes as the coupled-dipole and coupledquadrupole modes. If the frequencies of the coupleddipole and coupled-quadrupole modes become equal, the threshold for a fast "mode-coupling" instability is reached.

Because fast instabilities involving many synchrotron modes may result when there is no restoring force for a perturbation of the bunch position or length [15], the mode-coupling model may be applicable only when the equilibrium phase and zero-frequency quadrupole Robinson instabilities are avoided. Thus, these instabilities are also considered in the coupled-mode analysis.

\section{E. Coupled-bunch instability}

When a harmonic cavity has a low value of $Q$ or is detuned far from the frequency $\nu \omega_{g}$, it may excite dipole coupled-bunch oscillations with longitudinal mode numbers of 1 or $M-1$ (i.e., \pm 1 ). This instability is described by Eq. (12) provided that the sum is over $n=M j \pm 1$ $(j=-\infty, \ldots, \infty)[15]$. Consequently, for $\nu \omega_{g} \gg \omega_{0}$, the oscillation frequency and damping rate approximately obey Eqs. (13) and (15), provided that $\left(\Omega \pm \omega_{0}\right)$ is substituted for $\Omega$ in the definitions of the sideband tuning angles $\phi_{1+}, \phi_{1-}, \phi_{2+}$, and $\phi_{2-}$.

\section{F. Landau damping}

To estimate whether Landau damping is overcome in a general synchrotron potential, we would like to compare the complex frequency shift of the $\mu_{\mathrm{o}}$ th mode, $\Delta \Omega \equiv$ $\Omega-i \alpha_{R}-\mu_{\mathrm{o}} \omega_{R}$, to a Landau damping rate that is proportional to the synchrotron frequency spread. For a 
dipole mode in a mostly quadratic synchrotron potential, the Landau damping rate is $0.78 \sigma_{\omega_{s}}[23,24]$, where the synchrotron frequency spread is given by [5]

$$
\sigma_{\omega_{s}}=\frac{\alpha^{2}\left(\sigma_{E} / E\right)^{2}}{\omega_{s}}\left|\frac{3 c}{\omega_{s}^{2}}-\left(\frac{3 b}{\omega_{s}^{2}}\right)^{2}\right|
$$

In a quartic synchrotron potential, Appendix A indicates that the Landau damping rate is $0.31 \sigma_{\omega_{s}}$, where

$$
\sigma_{\omega_{s}}=1.17\left(U_{\mathrm{o}} c\right)^{1 / 4}
$$

yielding a Landau damping rate of $0.36\left(U_{\mathrm{o}} c\right)^{1 / 4}$. An approximate formula for the dipole Landau damping rate in a general synchrotron potential is given by

$$
|\Delta \Omega|_{\text {thresh }}=0.78 \frac{\alpha^{2}\left(\sigma_{E} / E\right)^{2}}{\omega_{R}}\left|\frac{3 c}{\omega_{R}^{2}}-\left(\frac{3 b}{\omega_{R}^{2}}\right)^{2}\right|
$$

For a mostly quadratic synchrotron potential $(\xi \ll 1)$, Eq. (19) is valid because $\omega_{R} \approx \omega_{s}$. For a quartic synchrotron potential $(\xi=1, b=0)$, Eq. (19) yields a Landau damping rate of $0.34\left(U_{0} c\right)^{1 / 4}$, which is $6 \%$ lower than that obtained with the Vlasov equation. Thus, Eq. (19) is expected to provide a good model of the dipole-mode Landau damping rate for $\xi \leq 1$.

For a quadrupole mode in a mostly quadratic potential, the Landau damping rate is $2.24 \sigma_{\omega_{s}}[23,24]$, while Appendix A indicates that, in a quartic potential, the Landau damping rate is $0.58 \sigma_{\omega_{s}}$. Assuming that the quadrupole Landau damping rate is $2.24 / 0.78$ times the dipole damping rate of Eq. (19) gives a valid result for $\xi \ll 1$. For a quartic synchrotron potential, a damping rate of $0.83 \sigma_{\omega_{s}}$ is thereby obtained, which is $43 \%$ high. A reasonable approximation of the quadrupole damping rate is thereby obtained for $\xi \leq 1$. An estimate of sextupole Landau damping may be obtained by multiplying the dipole damping rate by $4.12 / 0.78$, while octupole Landau damping may be estimated by multiplying the dipole damping rate by $6.36 / 0.78$ [24].

\section{PASSIVE LANDAU CAVITY WITH THE ALADDIN LATTICE}

\section{A. Analytic modeling}

To analyze a passive Landau cavity, we have modified an algorithm described in Ref. [5] to consider Robinson instabilities for a given fundamental rf voltage $V_{T 1}$, ring current $I$, and harmonic cavity tuning angle $\phi_{2}$. This analysis also calculates whether resonant interaction with a real parasitic impedance $Z\left(\omega_{C B}\right)$ at frequency $\sim \omega_{C B}$ will excite a dipole coupled-bunch instability, where the values of $Z\left(\omega_{C B}\right)$ and $\omega_{C B}$ are inputs to the analysis. Thus, one may estimate whether sufficient Landau damping is provided by the harmonic cavity to suppress parasitic coupled-bunch instabilities. The parameters used in modeling the standard Aladdin lattice and the LF20 lowemittance lattice are shown in Table I. The values of
TABLE I. Standard Aladdin parameters, with low-emittance (LF20) values of $V_{T 1}, \alpha$, and $\tau_{L}$ in parentheses.

\begin{tabular}{cc}
\hline \hline Parameter & Value \\
\hline$E$ & $800 \mathrm{MeV}$ \\
$\sigma_{E} / E$ & $4.8 \times 10^{-4}$ \\
$V_{s}$ & $17.4 \mathrm{kV}$ \\
$T_{\mathrm{o}}$ & $2.96 \times 10^{-7} \mathrm{~s}$ \\
$\omega_{g} / 2 \pi$ & $50.6 \mathrm{MHz}$ \\
$M$ & $15 \mathrm{bunches}$ \\
$R_{1}^{\mathrm{o}}$ (unloaded) & $0.5 \mathrm{M} \Omega$ \\
$Q_{1}^{\mathrm{o}}$ (unloaded) & 8000 \\
$\beta_{1}$ & 11 \\
$\nu$ & 4 \\
$R_{2}^{\mathrm{o}}$ (unloaded) & $1.24 \mathrm{M} \Omega$ \\
$Q_{2}^{\mathrm{o}}($ unloaded $)$ & 20250 \\
$\beta_{2}($ passive $)$ & 0 \\
$\omega_{C B} / 2 \pi$ & $1 \mathrm{GHz}$ \\
$Z\left(\omega_{C B}\right)$ & $10 \mathrm{k} \Omega$ \\
$V_{T 1}$ & $90 \mathrm{kV}(50 \mathrm{kV})$ \\
$\alpha$ & $0.0335(0.0043)$ \\
$\tau_{L}$ & $13.8 \mathrm{~ms}(13.5 \mathrm{~ms})$ \\
\hline \hline
\end{tabular}

$Z\left(\omega_{C B}\right)$ and $\omega_{C B}$ represent a typical higher-order mode of the Aladdin fundamental rf cavity.

We initially set $F_{1}=1$ and $F_{2}=0.1$, and iterate until the form factors are consistent with the computed bunch length. Our algorithm proceeds as follows.

(i) Calculate $\psi_{1}$, the synchronous phase (with $0^{\circ}<$ $\psi_{1}<90^{\circ}$ ), using the equation

$$
\begin{aligned}
V_{s} & =V_{T 1} \cos \psi_{1}+V_{T 2} \cos \psi_{2} \\
& =V_{T 1} \cos \psi_{1}-2 I R_{2} F_{2} \cos ^{2} \phi_{2} .
\end{aligned}
$$

If this equation can only be solved with $\left|\cos \psi_{1}\right|>1$, there is no equilibrium phase and the calculation is discontinued.

(ii) Calculate the tuning angle of Cavity 1 for operation in the compensated condition in which the beam-loaded cavity appears as a resistive load to the $\mathrm{rf}$ generator. In this case, $0^{\circ}<\phi_{1}<90^{\circ}$, and $\phi_{1}$ obeys $[19,20]$

$$
\phi_{1}=\tan ^{-1}\left(2 F_{1} I R_{1} \sin \psi_{1} / V_{T 1}\right) .
$$

(iii) Calculate the coefficients of the Taylor expansion of the synchrotron potential, $U(\tau)=a \tau^{2}+b \tau^{3}+$ $c \tau^{4}$, using Eqs. (4) $-(6)$, in which, for a passive Landau cavity, $V_{T 2} \cos \psi_{2}=-2 I F_{2} R_{2} \cos ^{2} \phi_{2} \quad$ and $V_{T 2} \sin \psi_{2}=I F_{2} R_{2} \sin 2 \phi_{2}$.

(iv) Calculate the bunch length using Eq. (8) with $U(\tau)=a \tau^{2}+b \tau^{3}+c \tau^{4}$.

(v) Calculate the form factors from the bunch lengths using the Gaussian bunch formulas $F_{1}=\exp \left(-\omega_{g}^{2} \sigma_{t}^{2} / 2\right)$ and $F_{2}=\exp \left(-\nu^{2} \omega_{g}^{2} \sigma_{t}^{2} / 2\right)$. We repeat steps (i) $-(\mathrm{v})$ if the newly computed form factors differ significantly from the values input at step (i). For new input values of $F_{1}$ and $F_{2}$, we use a weighted average of the newly computed form factors and their previous values. After several iterations, the form factors are consistent with the bunch length. 
(vi) Estimate whether the dipole longitudinal coupledbunch instability is damped for resonant interaction with a parasitic longitudinal cavity mode of impedance $Z\left(\omega_{C B}\right)$ at approximate frequency $\omega_{C B}$. For resonant interaction with a parasitic cavity mode, the complex frequency shift is imaginary with magnitude equaling the coupled-bunch growth rate, given by

$$
\left|\Delta \Omega_{C B}\right|=\frac{e I \alpha \omega_{C B} Z\left(\omega_{C B}\right) F_{\omega_{C B}}^{2}}{2 E T_{\mathrm{o}} \omega_{R}},
$$

where $F_{\omega_{C B}}$ is the bunch form factor at frequency $\omega_{C B}$. If, after subtracting the radiation damping rate $\tau_{L}^{-1}$, the growth rate remains positive, it is compared with the calculated dipole Landau damping rate of Eq. (19) to estimate whether Landau damping is sufficient to prevent instability.

We now consider Robinson instabilities.

(vii) To analyze Robinson stability without consideration of mode coupling, the single-mode formulas of Sec. II C are applied. For the $\mu_{\mathrm{o}}$ th mode (where $\mu_{\mathrm{o}}=1$, 2,3 , or 4 ), the zero-frequency instability is first considered. If the zero-frequency instability is not predicted, the frequency and Robinson damping rates (including radiation damping) are then calculated. If the resultant damping rate is negative (corresponding to growth), the magnitude of the complex frequency shift is compared with the Landau damping rate to predict whether Landau damping is overcome.

(viii) Next, we compute the frequency and damping rate of the dipole coupled-bunch modes with longitudinal mode numbers of \pm 1 , according to Sec. IIE. We add $\tau_{L}^{-1}$ to the damping rate to account for radiation damping. If the damping rate is negative, implying growth, we compare the frequency shift magnitude with the calculated dipole Landau damping rate to estimate whether Landau damping is overcome.

(ix) To include the effects of dipole-quadrupole mode coupling, the computation of dipole and quadrupole mode frequencies and damping rates is replaced by computation of the coupled-dipole and coupled-quadrupole Robinson frequencies and damping rates using Eqs. (B11)-(B13), which include radiation damping. To estimate whether the coupled-dipole mode overcomes Landau damping, we use the dipole-mode criterion. To estimate whether the coupled-quadrupole mode overcomes Landau damping, we use the quadrupole-mode criterion. Because a restoring force for slow dipole and quadrupole motions may be necessary for the dipole-quadrupole mode coupling theory to apply, we also test for the equilibrium phase and zero-frequency quadrupole instability thresholds.

We solve Eqs. (B11) and (B13) by iteration, starting with the values $\left(\Omega, \alpha_{R}\right)=\left(\omega_{R}, \tau_{L}^{-1}\right)$ for the coupleddipole mode and $\left(\Omega, \alpha_{R}\right)=\left(2 \omega_{R}, 2 \tau_{L}^{-1}\right)$ for the coupledquadrupole mode. To stabilize the iteration, we take an absolute value before performing the square root in Eq. (B13). If the argument of the square root is negative in the converged solution, a fast mode-coupling instability is predicted. We have confirmed that this method identifies the threshold in which the coupled-dipole and coupled-quadrupole mode frequencies are equal.

In Fig. 1(a), uncoupled dipole and quadrupole Robinson instabilities are predicted for the Aladdin lattice with a passive fourth harmonic cavity. The analysis is performed
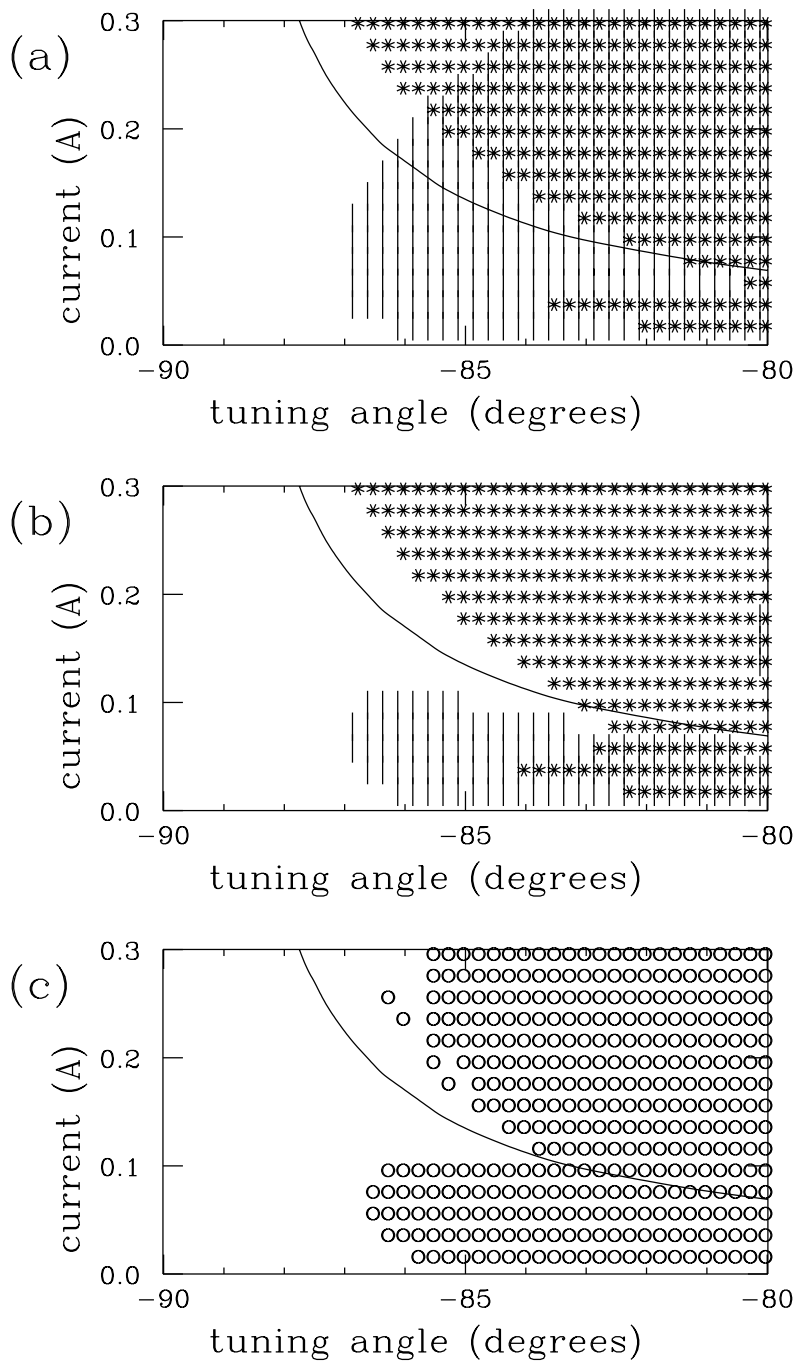

FIG. 1. (a) Robinson instabilities are predicted without consideration of mode coupling, for passive Landau-cavity operation with the Aladdin lattice and a fundamental rf voltage of $90 \mathrm{kV}$. A solid curve shows the parameters for optimal bunch lengthening, in which case the linear synchrotron frequency is zero. A symbol is plotted when Robinson instability is predicted for a given ring current and Landau-cavity tuning angle. Vertical line: dipole instability; *: quadrupole instability. (b) Dipole-quadrupole mode coupling is included in Robinson instability predictions. In this case, the dipole and quadrupole modes are perturbed by the coupling, but a fast mode-coupling instability is not predicted. Vertical line: coupled-dipole instability; * : coupled-quadrupole instability. (c) Instabilities observed in 500000 turn simulations. When the relative energy spread at the end of a simulation exceeds the natural value $\left(4.8 \times 10^{-4}\right)$ by $\geq 10 \%$, a circle is plotted to signify instability. 
for currents that are multiples of $20 \mathrm{~mA}$ and Landaucavity tuning angles that are multiples of $0.25^{\circ}$. A solid curve shows the tuning angle for optimal bunch lengthening $(\xi=1)$ versus ring current. For $\xi=0$, the Robinson angular frequency $\omega_{R}=62900 \mathrm{rad} / \mathrm{s} \approx \omega_{s} \quad\left(f_{R} \equiv\right.$ $\omega_{R} / 2 \pi=10000 \mathrm{~Hz}$ ), while, for $\xi=1, \quad \omega_{R}=$ $28600 \mathrm{rad} / \mathrm{s}\left(f_{R}=4550 \mathrm{~Hz}\right)$. When tuning in the cavity with currents below $180 \mathrm{~mA}$, a dipole Robinson instability is predicted to onset before optimal bunch lengthening is attained. For currents exceeding $200 \mathrm{~mA}$, a quadrupole Robinson instability is predicted to occur when the Landau cavity voltage is $15 \%$ higher than optimal $(\xi \approx 1.15)$. The sextupole Robinson instability is predicted to occur where the quadrupole Robinson instability is already present, in the upper right-hand corner of the plot where $I>140 \mathrm{~mA}$ and $\phi_{2}>-83^{\circ}$. Because the calculated quadrupole mode frequency $(\sim 11 \mathrm{kHz})$ exceeds that of the dipole mode $(\sim 10 \mathrm{kHz})$ by only $\sim 10 \%$ in the region of Fig. 1(a) where $0.9<\xi<1.15$, the uncoupled mode analysis may be inapplicable for optimally lengthened bunches.

In Fig. 1(b), dipole-quadrupole mode coupling is included in the analysis. When tuning in the Landau cavity at currents below $120 \mathrm{~mA}$, the coupled-dipole Robinson instability is predicted to onset well before optimal bunch lengthening is obtained. When tuning in the Landau cavity at higher currents, the coupled-quadrupole Robinson instability is predicted to onset after optimal bunch lengthening is obtained, when the Landau cavity voltage is $\sim 15 \%$ higher than required for optimal bunch lengthening.

\section{B. Simulation}

To study the evolution of unstable behavior, simulations have been performed in which 900 macroparticles are evenly distributed among the 15 buckets of Aladdin. The macroparticles of the $n$th bunch initially pass through the rf cavities at time $t=n t_{g}$ with energy equaling the synchronous energy, where $t_{g}=2 \pi / \omega_{g}$ is the time between buckets. On subsequent revolutions, random energy offsets of the macroparticles are generated by "synchrotron radiation"; these offsets then decay away from radiation damping. Passage of the macroparticles through the rf cavities results in energy gain or loss (depending upon the time of passage) and excitation of wake fields.

To speed up the simulations, the rf fields are described using slowly varying "in-phase" components $\left[V_{c 1}(t)\right.$ and $\left.V_{c 2}(t)\right]$ and "quadrature" components $\left[V_{s 1}(t)\right.$ and $\left.V_{s 2}(t)\right]$ which yield the total $\mathrm{rf}$ voltages in each cavity according to $[25,26]$

$$
\Delta V_{b c 1}=\frac{q}{2}\left[\frac{2 R_{1}}{\tau_{1}} \cos \left(\omega_{g} t_{m}\right)+\frac{R_{1}}{Q_{1} \tau_{1}} \sin \left(\omega_{g} t_{m}\right)\right]
$$

$$
\begin{aligned}
& V_{1}(t)=V_{c 1}(t) \cos \left(\omega_{g} t\right)-V_{s 1}(t) \sin \left(\omega_{g} t\right), \\
& V_{2}(t)=V_{c 2}(t) \cos \left(\nu \omega_{g} t\right)-V_{s 2}(t) \sin \left(\nu \omega_{g} t\right) .
\end{aligned}
$$

Here, $V_{c 1}(t)=V_{g c 1}+V_{b c 1}(t)$ is the sum of the constant rf-generator in-phase voltage $V_{g c 1}$ and that resulting from the beam's wake $V_{b c 1}(t)$. Similarly, $V_{s 1}(t), V_{c 2}(t)$, and $V_{s 2}(t)$ are sums of rf-generator voltages and wakes. For a passive Landau cavity, $V_{g c 2}=V_{g s 2}=0$. Within a single bunch, we neglect the time variation of the in-phase and quadrature rf-field components, except for that resulting from the accumulation of wake fields from each passing macroparticle. This is valid for $\mathrm{rf}$ cavities with $Q \gg 1$, provided that the macroparticles remain bunched within their rf buckets.

For a given ring current $I$, fundamental rf voltage $V_{T 1}$, and Cavity 2 tuning angle $\phi_{2}$, we first perform steps (i)-(v) of Sec. III A to determine the synchronous phase $\psi_{1}$, form factors $F_{1}$ and $F_{2}$, and Cavity 1 tuning angle $\phi_{1}$. Steady-state operation has $V_{c 1}=V_{T 1} \cos \psi_{1}$, $V_{s 1}=V_{T 1} \sin \psi_{1}$, with beam-loading contributions $V_{b c 1}=$ $-2 F_{1} I R_{1} \cos ^{2} \phi_{1}, V_{b s 1}=F_{1} I R_{1} \sin 2 \phi_{1}$, so that the rfgenerator in-phase and quadrature voltages are given by

$$
\begin{aligned}
& V_{g c 1}=V_{T 1} \cos \psi_{1}+2 F_{1} I R_{1} \cos ^{2} \phi_{1}, \\
& V_{g s 1}=V_{T 1} \sin \psi_{1}-F_{1} I R_{1} \sin 2 \phi_{1} .
\end{aligned}
$$

The resonant frequencies of the rf cavities are determined from the tuning angles by

$$
\begin{aligned}
& \omega_{1}=\omega_{g} /\left(1+\tan \phi_{1} / 2 Q_{1}\right), \\
& \omega_{2}=\nu \omega_{g} /\left(1+\tan \phi_{2} / 2 Q_{2}\right),
\end{aligned}
$$

while the rf decay times are

$$
\tau_{1}=2 Q_{1} / \omega_{1}, \quad \tau_{2}=2 Q_{2} / \omega_{2} .
$$

We now track macroparticles. When the $m$ th macroparticle belongs to the $n$th bunch, let $t_{m}$ denote its arrival time at the rf cavities relative to the reference time $n t_{g}$, while its energy offset relative to the synchronous energy is denoted $\varepsilon_{m}$. We begin our simulation with $t_{m}=\varepsilon_{m}=0$ for all macroparticles, and no wake fields $\left[V_{b c 1}(0)=V_{b s 1}(0)=\right.$ $\left.V_{b c 2}(0)=V_{b s 2}(0)=0\right]$. Physically, this corresponds to injecting cold bunches at the synchronous phase during a single revolution.

The macroparticles within a bunch are considered one at a time, starting with the one that arrives earliest. According to the fundamental theorem of beam loading, 1/2 of each macroparticle's wake is added to the cavity fields before computing its energy gain or loss; the remaining half of the wake is added afterwards. One-half of the wake from the macroparticle charge $q=-T_{\mathrm{o}} I / 900$ causes the Cavity 1 wake voltage to change at the reference time $n t_{g}$ by [26]

$$
\Delta V_{b s 1}=\frac{q}{2}\left[\frac{R_{1}}{Q_{1} \tau_{1}} \cos \left(\omega_{g} t_{m}\right)-\frac{2 R_{1}}{\tau_{1}} \sin \left(\omega_{g} t_{m}\right)\right] .
$$


Similarly, the Cavity 2 wake voltage at the reference time is changed by

$$
\Delta V_{b c 2}=\frac{q}{2}\left[\frac{2 R_{2}}{\tau_{2}} \cos \left(\nu \omega_{g} t_{m}\right)+\frac{R_{2}}{Q_{2} \tau_{2}} \sin \left(\nu \omega_{g} t_{m}\right)\right], \quad \Delta V_{b s 2}=\frac{q}{2}\left[\frac{R_{2}}{Q_{2} \tau_{2}} \cos \left(\nu \omega_{g} t_{m}\right)-\frac{2 R_{2}}{\tau_{2}} \sin \left(\nu \omega_{g} t_{m}\right)\right] .
$$

When $t_{m}>0$, the wake voltage increments of Eqs. (27) and (28) are fictitious because the reference time $n t_{g}$ precedes the macroparticle's passage. However, the incremented wake voltage is used only to compute the wake at times $\geq t_{m}$, so that no fictitious effects arise in the tracking.

For a natural energy spread of $\sigma_{E}$ and radiation damping time $\tau_{L}$, the macroparticle energy offset is incremented by $[27,28]$

$$
\begin{aligned}
\Delta \varepsilon_{m}= & \left(-2 T_{\mathrm{o}} / \tau_{L}\right) \varepsilon_{m}+2 \sigma_{E}\left(T_{\mathrm{o}} / \tau_{L}\right)^{1 / 2} R_{m n}+e\left[V\left(t_{m}\right)-V_{s}\right] \\
= & \left(-2 T_{\mathrm{o}} / \tau_{L}\right) \varepsilon_{m}+2 \sigma_{E}\left(T_{\mathrm{o}} / \tau_{L}\right)^{1 / 2} R_{m n} \\
& +e\left[V_{g c 1} \cos \left(\omega_{g} t_{m}\right)-V_{g s 1} \sin \left(\omega_{g} t_{m}\right)+V_{g c 2} \cos \left(\nu \omega_{g} t_{m}\right)-V_{g s 2} \sin \left(\nu \omega_{g} t_{m}\right)\right. \\
& \left.\quad+V_{b c 1} \cos \left(\omega_{g} t_{m}\right)-V_{b s 1} \sin \left(\omega_{g} t_{m}\right)+V_{b c 2} \cos \left(\nu \omega_{g} t_{m}\right)-V_{b s 2} \sin \left(\nu \omega_{g} t_{m}\right)-V_{s}\right],
\end{aligned}
$$

where $R_{m n}$ is a random number with zero mean and unity standard deviation. For a passive Landau cavity, we have $V_{g c 2}=$ $V_{g s 2}=0$ in Eq. (29). The remaining half of the macroparticle's wake field is now included by executing Eqs. (27) and (28) again. The macroparticle time offset is incremented by

$$
\Delta t_{m}=\alpha T_{\mathrm{o}} \varepsilon_{m} / E
$$

We then consider the remaining macroparticles in the bunch.

After considering all of the macroparticles within a bunch, the Cavity 1 wake fields are propagated ahead one rf period to the new reference time $(n+1) t_{g}$ using [26]

$$
\begin{aligned}
& V_{b c 1}\left[(n+1) t_{g}\right]=\exp \left(-t_{g} / \tau_{1}\right)\left[\cos \left(\Delta \omega_{1} t_{g}\right) V_{b c 1}\left(n t_{g}\right)-\sin \left(\Delta \omega_{1} t_{g}\right) V_{b s 1}\left(n t_{g}\right)\right] \\
& V_{b s 1}\left[(n+1) t_{g}\right]=\exp \left(-t_{g} / \tau_{1}\right)\left[\cos \left(\Delta \omega_{1} t_{g}\right) V_{b s 1}\left(n t_{g}\right)+\sin \left(\Delta \omega_{1} t_{g}\right) V_{b c 1}\left(n t_{g}\right)\right]
\end{aligned}
$$

where $\Delta \omega_{1} \equiv \omega_{1}-\omega_{g}$. The Cavity 2 wake fields are propagated by the same equation with subscripts " 1 " changed to " 2 " and $\Delta \omega_{2} \equiv \omega_{2}-\nu \omega_{g}$. The macroparticle tracking of the next bunch may now be performed. As beam diagnostics, we calculate the average and rms values $\langle t\rangle,\langle\varepsilon\rangle, \sigma_{t}$, and $\sigma_{\varepsilon}$ for each turn, in addition to the distributions of $t_{m}$ and $\varepsilon_{m}$ after the final turn that is tracked. We have typically tracked for 500000 turns $(0.148 \mathrm{~s})$, which is $\sim 11$ times the radiation damping time.

In our simulations, the energy spread $\sigma_{\varepsilon}$ increases above its natural value when oscillations of the bunch centroid $\langle t\rangle$ or length $\sigma_{t}$ indicate instability, or in cases where electrons are not confined to their rf buckets. Because such cases generally occur within a regime of strong instability, they may also be taken to signify instability. Figure 1(c) shows simulation results for a passive Landau cavity in which a circle indicates that the average energy spread during the final 10000 turns exceeds its natural value by more than $10 \%$. The simulation results are in approximate agreement with the analytic predictions of Fig. 1(b) in which dipolequadrupole coupling is included.

The simulated instability growth and saturation for a current of $100 \mathrm{~mA}$ and $\phi_{2}=-86^{\circ}$ is shown in Fig. 2. The amplitude of the beam centroid oscillations greatly exceeds that of the oscillations in $\sigma_{t}$, indicating a dipolelike instability. From the simulation, an oscillation frequency of $9900 \mathrm{~Hz}$ and exponential growth rate of $95 \mathrm{~s}^{-1}$ were determined. This agrees approximately with the analytic prediction that the coupled dipole mode is unstable, with frequency equaling $10000 \mathrm{~Hz}$ and growth rate of $70 \mathrm{~s}^{-1}$.

Figure 3 shows the simulated unstable behavior for a current of $200 \mathrm{~mA}$ when $\phi_{2}=-85^{\circ}$, showing a relaxation oscillation in $\sigma_{t}$. The amplitude of $\sigma_{t}$ oscillations is much greater than that of the beam centroid, consistent with analytic prediction of a coupled-quadrupole instability. The simulated oscillation frequency of $14.4 \mathrm{kHz}$ is $8 \%$ higher than the analytic calculation of $13.3 \mathrm{kHz}$, within the expected $\sim 10 \%$ accuracy of the analytic calculations for

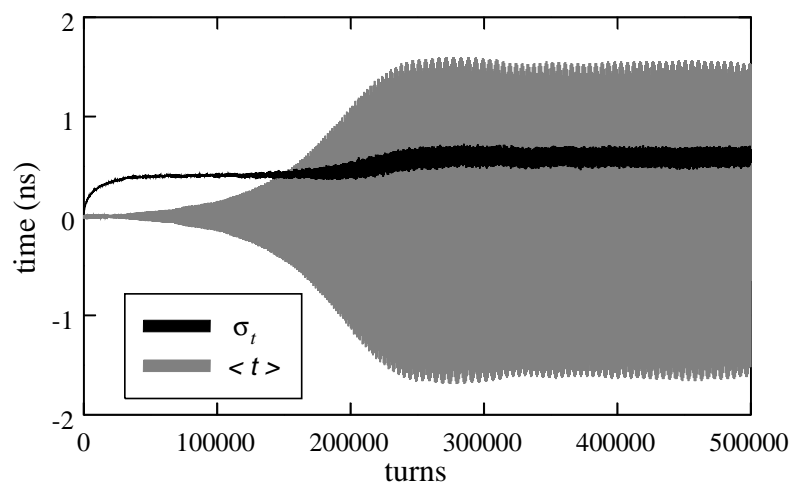

FIG. 2. Simulation of coupled-dipole Robinson instability for passive Landau-cavity operation with the Aladdin lattice, for a ring current of $100 \mathrm{~mA}$ and Landau-cavity tuning angle of $-86^{\circ}$. The time offset of the bunch centroid $\langle t\rangle$ and bunch length $\sigma_{t}$ are plotted every 100 turns. 


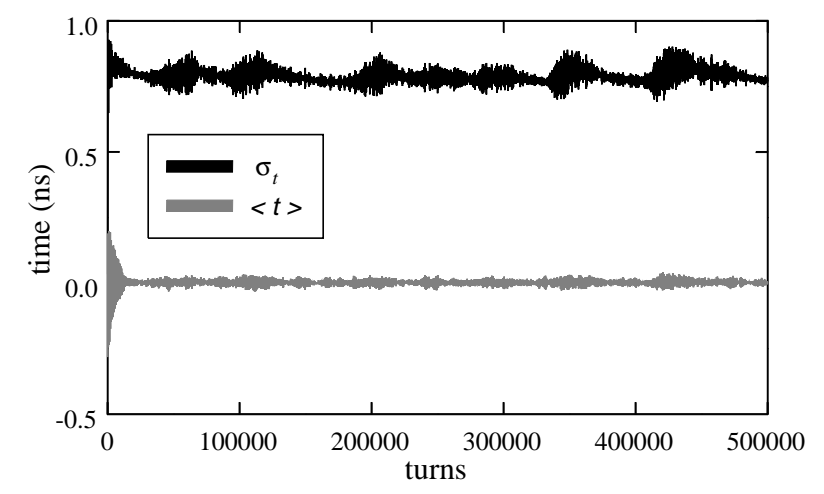

FIG. 3. Simulation of coupled-quadrupole Robinson instability for passive Landau-cavity operation with the Aladdin lattice, for a ring current of $200 \mathrm{~mA}$ and Landau-cavity tuning angle of $-85^{\circ}$.

$\xi \sim 1$. An exponential growth rate could not be estimated from the simulated relaxation oscillations.

\section{Experiment}

Experimental results were obtained by varying the tuning angle of the Aladdin harmonic cavity at a fixed beam current. The bunch shape and length were obtained from a capacitive pickup [6] whose time response broadens the bunch length measurements by $\sim 100$ ps. For single-hump bunches, a computer program fits the measured data to a Gaussian bunch shape to yield a bunch length. An uncalibrated voltage monitor in the harmonic cavity was used to estimate $\xi$ within $\sim 5 \%$ by assuming that $\xi$ is proportional to voltage and equals 1 at the dividing line between single-hump and double-hump bunches.

Without the Landau cavity, synchrotron sidebands were observed on a tune signal by slightly turning down the sextupoles. They were separated from the main tune signal by $10 \mathrm{kHz}$, in agreement with the analytic prediction that $f_{R} \equiv \omega_{R} / 2 \pi=10 \mathrm{kHz}$.

Experiments with ring currents of 113 and $213 \mathrm{~mA}$ were compared with analytic results and simulations for 100 and $200 \mathrm{~mA}$. For $I=113$ and $213 \mathrm{~mA}$, parasitic coupledbunch instabilities were suppressed experimentally when $\xi$ exceeded 0.3 , within $8 \%$ of analytic estimates of the threshold values of $\xi$. For $I=113 \mathrm{~mA}$, an apparent Robinson instability onset when $\xi=0.78$, within $10 \%$ of the predicted value of $\xi$ for onset of coupled-dipole Robinson instability. For $I=213 \mathrm{~mA}$, an apparent Robinson instability onset when $\xi=1.09$, agreeing with the analytic prediction for the coupled-quadrupole Robinson instability within $5 \%$.

In Fig. 4, we display experimental measurements of the bunch length versus $\xi$ taken with $I=213 \mathrm{~mA}$, as well as the analytic result of Eq. (8) and values from simulations. The three curves agree within the $\sim 100$ ps experimental uncertainty. With optimal bunch lengthening $(\xi=1)$, the bunch length is approximately doubled [6].

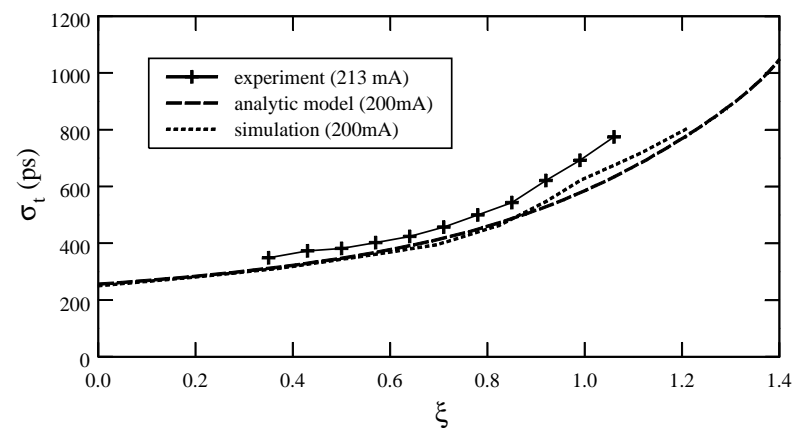

FIG. 4. Bunch length determined from experiment, analytic modeling, and simulations, for passive Landau-cavity operation with the Aladdin lattice. The parameter $\xi$ is proportional to the Landau-cavity voltage, equaling unity for an optimally lengthened bunch.

The analytic modeling of coupled dipole-quadrupole modes, simulations, and experiments agree for passive operation of the harmonic cavity with the standard Aladdin lattice. Because of Robinson instability, optimal bunch lengthening with passive operation of the harmonic cavity is limited to currents exceeding $\sim 120 \mathrm{~mA}[6]$.

\section{PASSIVE LANDAU CAVITY WITH THE LOW-EMITTANCE LF20 LATTICE}

\section{A. Analytic modeling}

The analytic results for passive harmonic-cavity operation with the LF20 lattice were obtained by changing three parameters input to the analysis. The momentum compaction was decreased from 0.0335 to 0.0043 , while the longitudinal radiation damping time changed slightly from 13.8 to $13.5 \mathrm{~ms}$. For comparison with experimental data taken with an rf voltage of $50 \mathrm{kV}$ (where maximum LF20 lifetime is achieved experimentally), the rf voltage was reduced from 90 to $50 \mathrm{kV}$.

In Fig. 5(a), instability predictions are made analytically for uncoupled dipole and quadrupole Robinson modes. Unstable sextupole and octupole modes are predicted for parameters where the quadrupole mode is also unstable, in the upper right-hand corner of the plot where $I>100 \mathrm{~mA}$ and $\phi_{2}>-82.5^{\circ}$. For $\xi=0$, the Robinson angular frequency $\omega_{R}=16450 \mathrm{rad} / \mathrm{s} \approx \omega_{s} \quad\left(f_{R} \equiv \omega_{R} / 2 \pi=\right.$ $2620 \mathrm{~Hz})$, while, for $\xi=1, \omega_{R}=5280 \mathrm{rad} / \mathrm{s}\left(f_{R}=\right.$ $840 \mathrm{~Hz}$ ). For currents exceeding $60 \mathrm{~mA}$, stability is predicted for optimally lengthened bunches. However, for $0.85<\xi<1.10$ (approximately), the calculated quadrupole mode frequency is less than the calculated dipole mode frequency of $\sim 2500 \mathrm{~Hz}$, so that the uncoupled-mode analysis may be invalid.

In Fig. 5(b), dipole-quadrupole mode coupling is included in the analysis. A fast mode-coupling instability is predicted for near-optimal bunch lengthening where $0.85<\xi<1.10$, while a large stable region is predicted for double-hump bunches with $\xi>1.10$ and $I>80 \mathrm{~mA}$. 

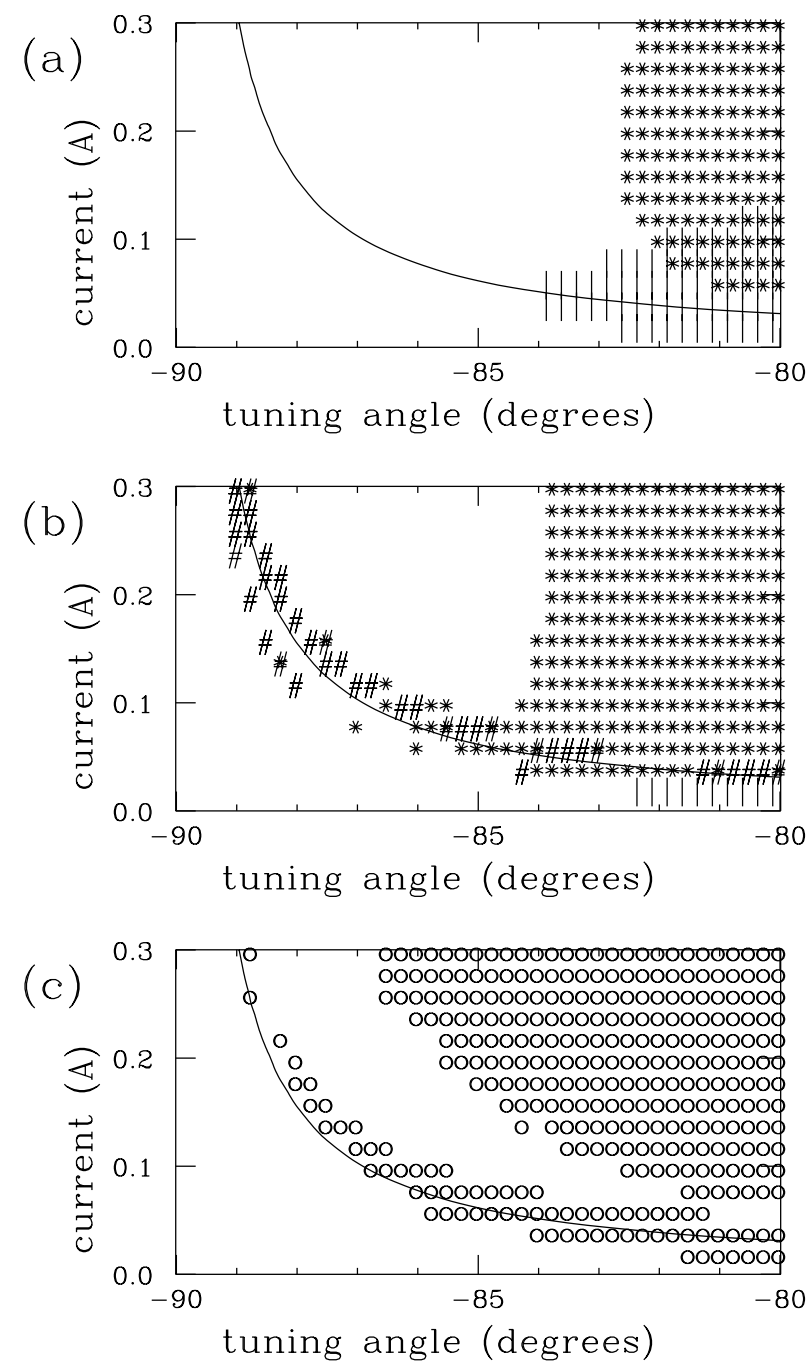

FIG. 5. Robinson instabilities are predicted without consideration of mode coupling, for passive Landau-cavity operation with the LF20 low-emittance lattice and a fundamental rf voltage of $50 \mathrm{kV}$. Vertical line: dipole instability; * : quadrupole instability. (b) Dipole-quadrupole mode coupling is included in Robinson instability predictions. Vertical line: coupled-dipole instability; * : coupled-quadrupole instability; \#: fast mode-coupling instability. (c) Instabilities observed in 500000 turn simulations.

\section{B. Simulations}

For passive harmonic cavity operation with the LF20 lattice, Fig. 5(c) shows the instabilities observed in simulations, where a final energy spread exceeding the natural value by more than $10 \%$ is taken to signify instability. The simulation results confirm the existence of a band of instability for optimally lengthened bunches with $\xi \approx 1$.

In simulations with $I=200 \mathrm{~mA}$ and $\xi \approx 1$, the amplitude of unstable oscillations is largest when $\phi_{2}=$ $-88.08^{\circ}$. Because of instability, the values of $\xi$ and $\sigma_{E} / E$ do not remain constant. At the end of the 500000 th turn, $\xi$ equals 1.14 , while the relative energy spread $\sigma_{E} / E$ equals $5.9 \times 10^{-4}, 23 \%$ larger than the natural energy spread of $4.8 \times 10^{-4}$. The simulation results, shown in Fig. 6 ,

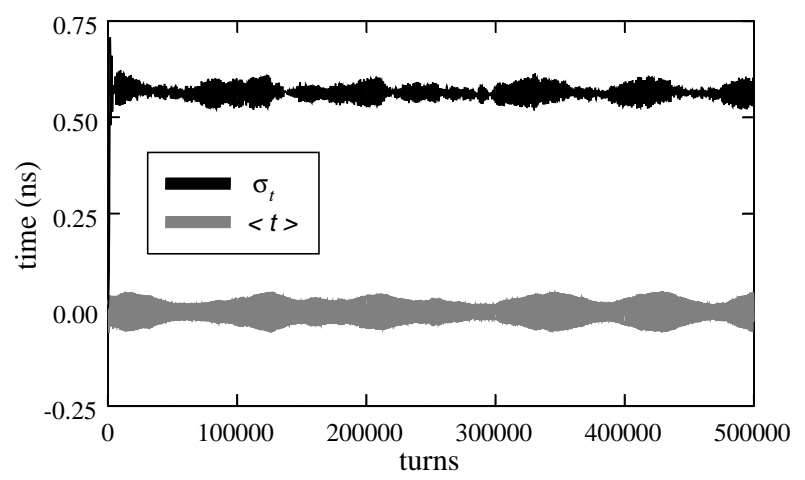

FIG. 6. Simulation of a fast mode-coupling Robinson instability for passive Landau-cavity operation with the LF20 lattice, for a ring current of $200 \mathrm{~mA}$ and Landau-cavity tuning angle of $-88.08^{\circ}$.

indicate that the bunch centroid position and bunch length undergo oscillations of comparable magnitude, suggesting a large degree of dipole-quadrupole mode coupling. Indeed, instability for these parameters is not predicted when coupling between dipole and quadrupole modes is neglected, while analytic modeling which includes dipole-quadrupole mode coupling predicts a fast modecoupling instability.

\section{Experiment}

Experiments were performed on the LF20 lattice with a fundamental $\mathrm{rf}$ voltage of $50 \mathrm{kV}$. Without the Landau cavity, synchrotron sidebands were observed on a tune signal by slightly turning down the sextupoles. They were separated from the main tune signal by $2.75 \mathrm{kHz}$, in approximate agreement with the analytic prediction that $f_{R} \equiv \omega_{R} / 2 \pi=2.6 \mathrm{kHz}$.

For $I=107$ and $220 \mathrm{~mA}$, parasitic coupled-bunch instabilities were suppressed experimentally for $\xi \geq 0.7$, in approximate agreement with the analytic estimates. This threshold value of $\xi$ is about twice as large as that measured with the standard Aladdin lattice and a $90 \mathrm{kV}$ rf voltage. For $I=107 \mathrm{~mA}$, Robinson instabilities were observed experimentally for $0.88<\xi<1.13$ and $\xi>1.23$. For $I=220 \mathrm{~mA}$, Robinson instabilities were observed experimentally for $1.01<\xi<1.15$ and $\xi>1.30$. The experimental observations confirm the existence of a band of instability for optimally lengthened bunches with $\xi \approx 1$, as did the simulations.

Figure 7(a) displays experimental measurements of the LF20 bunch length versus harmonic cavity voltage (parametrized by $\xi$ ), for ring currents of 107 and $220 \mathrm{~mA}$. The time response of the capacitive pickup broadens the measurements by $\sim 100$ ps. For $\xi \leq 1$, the bunch lengths are given by a Gaussian fit to the bunch shape, while the double-hump bunch length for $\xi=1.2$ was determined from an oscilloscope trace. Analytic modeling and simulations give nearly identical results for $I=100$ and $200 \mathrm{~mA}$; results for $I=200 \mathrm{~mA}$ are plotted. 

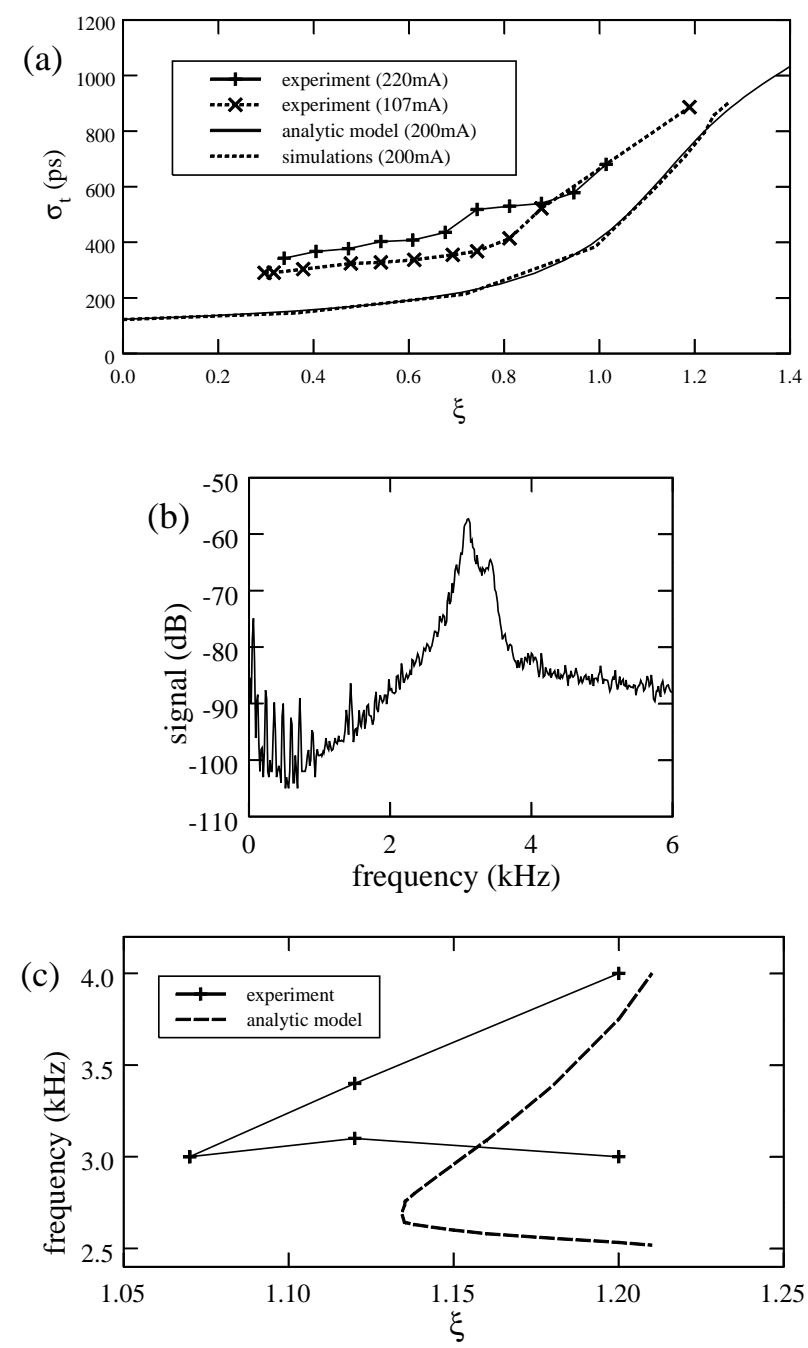

FIG. 7. (a) Bunch length determined from experiment, analytic modeling, and simulations, for passive Landau-cavity operation with the LF20 lattice. The parameter $\xi$ is proportional to the Landau-cavity voltage, equaling unity for an optimally lengthened bunch. (b) Bunch oscillation signal for passive Landaucavity operation with the LF20 lattice and a ring current of $128 \mathrm{~mA}$, for a stable double-hump bunch with $\xi=1.12$. Peaks are observed at 3.1 and $3.4 \mathrm{kHz}$. (c) Bunch oscillation frequencies observed for passive Landau-cavity operation with the LF20 lattice at a ring current of $128 \mathrm{~mA}$. Instability occurs when the frequencies converge for $\xi=1.07$. Analytic modeling of the coupled-dipole and coupled-quadrupole mode frequencies is also shown.

For $\xi \ll 1$, the measured $200 \mathrm{~mA}$ bunch length is larger than the measured $100 \mathrm{~mA}$ bunch length. This is an apparent effect of the microwave instability caused by the ring's broadband impedance (estimated to be in the range of 10-15 $\Omega$ ), in which the energy spread increases with current. Measurements of the beam's horizontal size suggest that the microwave instability increases the energy spread above its natural value for currents exceeding $\sim 60 \mathrm{~mA}$ without the Landau cavity and for currents exceeding $\sim 180 \mathrm{~mA}$ when a long bunch is obtained with the
Landau cavity. Because the ring's broadband impedance is poorly characterized, it is not included in the analytic modeling or simulations.

In Fig. 7(b), we display a bunch oscillation signal obtained by connecting a spectrum analyzer to a beam phase detector circuit, for the case of a stable double-hump bunch with $I=128 \mathrm{~mA}$ and $\xi=1.12$. When taking the data of Figs. 7(b) and 7(c), an rf circulator was attached to the Landau cavity coupling loop, so that $\beta_{2} \approx 1.5$. We observe two peaks, which may be interpreted as frequencies of collective oscillations excited by noise. The frequencies of these peaks were observed as a function of $\xi$ by varying the Landau cavity tuning angle. The frequencies converge when $\xi$ equals 1.07 , at which point instability increases the oscillation amplitude by $25 \mathrm{~dB}$ (a factor of 18). In Fig. 7(c), the observed frequencies are compared with analytic calculations of the coupled-dipole and coupled-quadrupole Robinson mode frequencies, showing approximate agreement. The data supports our interpretation of instability resulting from dipole-quadrupole mode coupling.

Approximate agreement between analytic modeling, simulations, and experiment is therefore obtained for passive operation of the harmonic cavity with the LF20 lattice, provided that dipole-quadrupole mode coupling is included in the analytic modeling.

\section{ACTIVE LANDAU CAVITY WITH THE ALADDIN LATTICE}

\section{A. Analytic modeling}

To analyze an active Landau cavity, we modified an algorithm described in Ref. [11]. The fast-rf feedback of the two cavities is included in the equivalent rf-coupling coefficients $\beta_{1}$ and $\beta_{2}$. For active operation of the harmonic cavity, the combination of a circulator and fast feedback is estimated to be approximately equivalent to an rfcoupling coefficient of $\beta_{2} \approx 160$. We consider operation of the rf cavities in the compensated condition $[19,20]$ with the rf-generator current in phase with the cavity voltage, for the case where the cubic term of the synchrotron potential is made equal to zero to produce an approximately symmetric bunch.

For a given ring current $I$, fundamental rf voltage $V_{T 1}$, and Landau cavity voltage parametrized by $\xi$, our algorithm proceeds as follows.

(i) Calculate $\psi_{1}$ and $\psi_{2}$, the synchronous phase angles of Cavities 1 and 2, and $V_{T 2}$, the peak voltage in Cavity 2. For a given value of $\xi$, we have

$$
V_{T 1} \sin \psi_{1}+(1 / \xi) \nu V_{T 2} \sin \psi_{2}=0
$$

The cubic term of the synchrotron potential is zero when

$$
V_{T 1} \cos \psi_{1}+\nu^{2} V_{T 2} \cos \psi_{2}=0
$$


The energy of a synchronous electron is unchanged by a revolution about the ring,

$$
V_{s}=V_{T 1} \cos \psi_{1}+V_{T 2} \cos \psi_{2} \text {. }
$$

From Eqs. (32)-(34), we obtain

$$
\begin{gathered}
\psi_{1}=\cos ^{-1}\left[\frac{V_{s}}{\left(1-\frac{1}{\nu^{2}}\right) V_{T_{1}}}\right], \\
\psi_{2}=\tan ^{-1}\left(\nu \xi \tan \psi_{1}\right)-180^{\circ},
\end{gathered}
$$
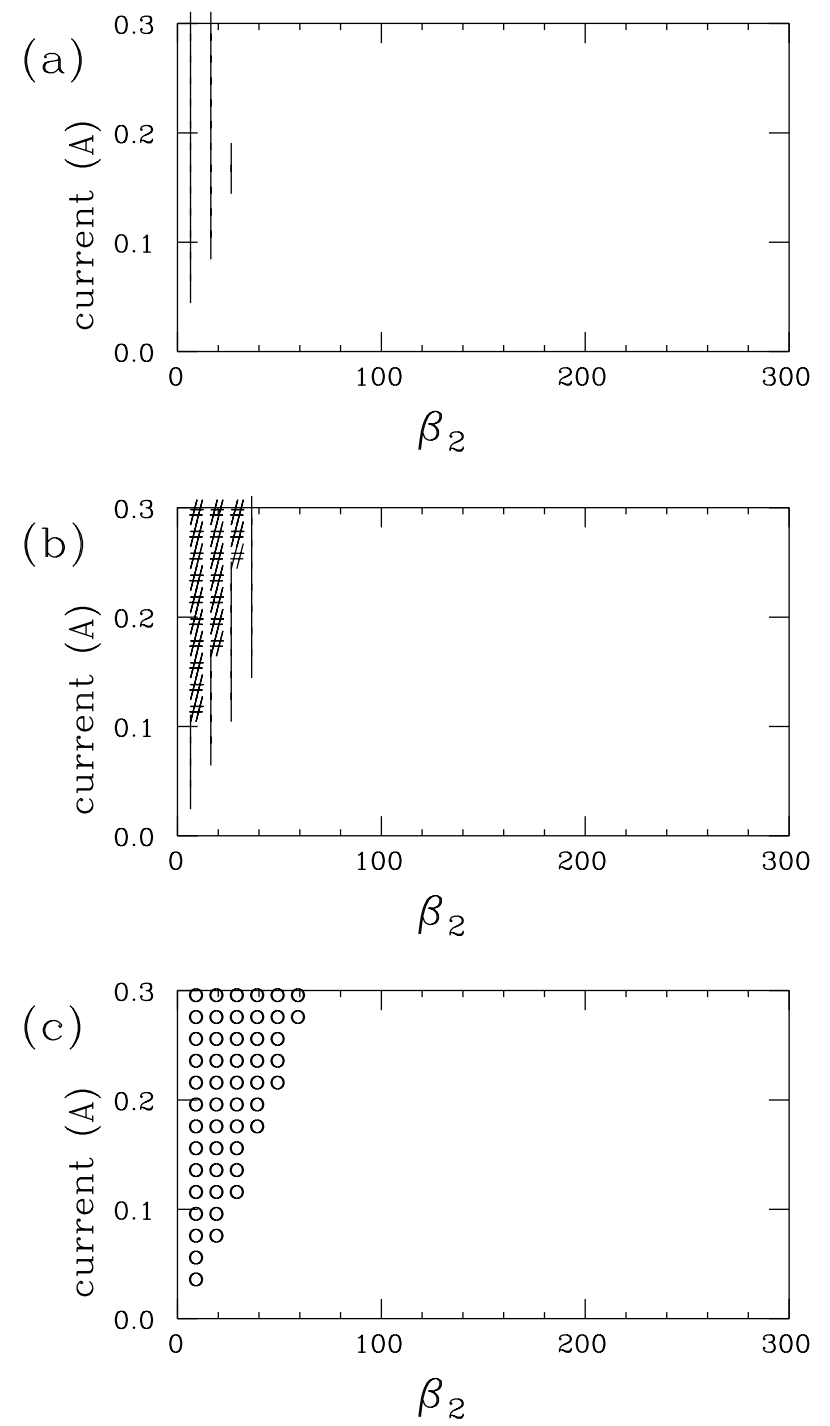

FIG. 8. (a) Robinson instabilities are predicted without consideration of mode coupling, for active Landau-cavity operation with optimally lengthened bunches in the Aladdin lattice with a fundamental rf voltage of $90 \mathrm{kV}$. A symbol is plotted when Robinson instability is predicted for a given ring current and effective rf coupling of the Landau cavity $\left(\beta_{2}\right)$. Vertical line: dipole instability. (b) Dipole-quadrupole mode coupling is included in Robinson instability predictions. Vertical line: coupled-dipole instability; \#: fast mode-coupling instability. (c) Instabilities observed in 500000 turn simulations. When the relative energy spread at the end of a simulation exceeds the natural value $\left(4.8 \times 10^{-4}\right)$ by $\geq 10 \%$, a circle is plotted to signify instability.

$$
V_{T 2}=-\xi V_{T 1} \sin \psi_{1} /\left(\nu \sin \psi_{2}\right) .
$$

If Eq. (35) cannot be solved with $\psi_{1}$ between $0^{\circ}$ and $90^{\circ}$, there is no equilibrium phase.

(ii) Calculate the quadratic and quartic coefficients of the synchrotron potential ( $a$ and $c$ ) using Eqs. (4) and (6).

(iii) Calculate the bunch length using Eq. (8) with $U(\tau)=a \tau^{2}+c \tau^{4}$, and the form factors using formulas for Gaussian bunches, $F_{1}=\exp \left(-\omega_{g}^{2} \sigma_{t}^{2} / 2\right)$ and $F_{2}=\exp \left(-\nu^{2} \omega_{g}^{2} \sigma_{t}^{2} / 2\right)$.

(iv) Calculate the tuning angle of the rf cavities for operation in the compensated condition

$$
\begin{aligned}
& \phi_{1}=\tan ^{-1}\left(2 F_{1} I R_{1} \sin \psi_{1} / V_{T 1}\right), \\
& \phi_{2}=\tan ^{-1}\left(2 F_{2} I R_{2} \sin \psi_{2} / V_{T 2}\right) .
\end{aligned}
$$

(v) We now analyze instabilities in the same manner as with a passive Landau cavity, described in steps (vi)-(ix) of Sec. III A.

In Fig. 8(a), uncoupled dipole and quadrupole Robinson instabilities are predicted for an optimally lengthened bunch $(\xi=1)$, for a variety of ring currents $I$ and Landaucavity coupling coefficients $\beta_{2}$. Figure $8(\mathrm{~b})$ displays predictions when coupling between dipole and quadrupole modes is included in the analysis. Stability is predicted for $\beta_{2}>40$. Nearly the same predictions were obtained for optimally lengthened bunches by using the quarticpotential formula for the coupled dipole-quadrupole instability [Eq. (B5)]. Simulation results (described below) are shown in Fig. 8(c).

To model Landau-cavity operation for a specified value of $\beta_{2}$ (i.e., a given feedback gain), dipole-quadrupole mode coupling is included in the analysis for a variety of Landau-cavity voltages (parametrized by $\xi$ ) and ring currents. In Fig. 9, we consider the case where $\beta_{2}=$ 160 . The loaded $Q$ is sufficiently low that the coupledbunch instability with longitudinal mode number equaling 1 is predicted to be excited by the harmonic cavity for

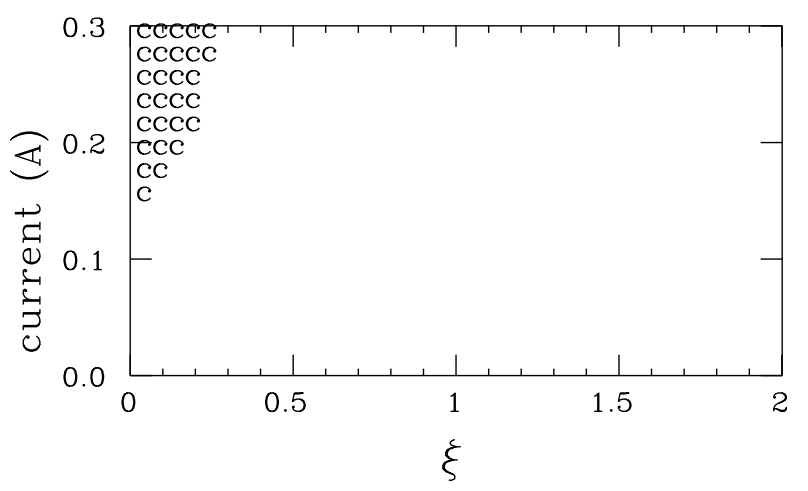

FIG. 9. Instability predictions which include dipolequadrupole mode coupling, for active Landau-cavity operation with the Aladdin lattice and $\beta_{2}=160$. c: coupled-bunch instability with longitudinal mode number of 1 . 
$I \geq 140 \mathrm{~mA}$ and $\xi<0.3$; stable operation is predicted when $\xi>0.3$.

\section{B. Simulations}

To simulate an active Landau cavity for a specified ring current $I$, fundamental $\mathrm{rf}$ voltage $V_{T 1}$, and value of $\xi$, we first perform steps (i)-(iv) of Sec. V A to determine the synchronous phases $\psi_{1}$ and $\psi_{2}$, form factors $F_{1}$ and $F_{2}$, tuning angles $\phi_{1}$ and $\phi_{2}$ (for operation in the compensated condition) and Landau cavity voltage $V_{T 2}$. The rfgenerator in-phase and quadrature voltages for Cavity 1 are then determined by Eq. (24); the rf-generator voltage components for Cavity 2 are determined by substituting the subscript "2" for "1" in Eq. (24). From this point on, the simulations proceed identically to those for a passive harmonic cavity.

An evaluation of $\xi$ and $V_{T 1}$ at the end of stable simulations indicates that the specified values are obtained within $\sim 3 \%$ except when $\xi \gg 1$, in which case the specified value of $\xi$ was obtained within $\sim 10 \%$. Thus, the rfgenerator voltage components are accurately obtained by the above method.

In Fig. 8(c), instabilities observed in simulations are shown for an optimally lengthened bunch $(\xi=1)$, for a variety of ring currents $I$ and Landau-cavity coupling coefficients $\beta_{2}$. The simulations agree approximately with the analytic results of Fig. 8(b).

Simulations were performed for $\beta_{2}=160$ with $I=$ 100,200 , and $300 \mathrm{~mA}$ and $0<\xi<2$. Consistent with the analytic predictions, no Robinson instabilities were observed. For $I=200 \mathrm{~mA}$, a coupled-bunch instability with mode number of \pm 1 was observed for $0.02 \leq \xi \leq 0.16$ while, for $I=300 \mathrm{~mA}$, this instability was observed for $0.008 \leq \xi \leq 0.29$, in excellent agreement with the analytic predictions of Fig. 9. A coupled-bunch instability with mode number of \pm 1 was identified in simulations by the sinusoidal dependence of bunch arrival time versus bunch number.

Simulations performed for $\beta_{2}$ equaling 20 and 40 displayed Robinson instabilities, in agreement with the analytic predictions of Fig. 8.

\section{Experiment}

Experimental studies of active harmonic cavity operation with the Aladdin lattice were performed for ring currents of 84 and $193 \mathrm{~mA}$, with an estimated value of $\beta_{2}=$ 160. Parasitic coupled-bunch instabilities were suppressed for $\xi \geq 0.21$ and $\xi \geq 0.33$, respectively. These threshold values of $\xi$ agree with the analytic predictions within $10 \%$.

In Fig. 10, the measured bunch length with $I=$ $193 \mathrm{~mA}$ is compared with analytic modeling and simulations performed for $I=200 \mathrm{~mA}$. The agreement is quite good, considering that the experimental determination of $\xi$ is accurate to within $\sim 5 \%$, while the experimental

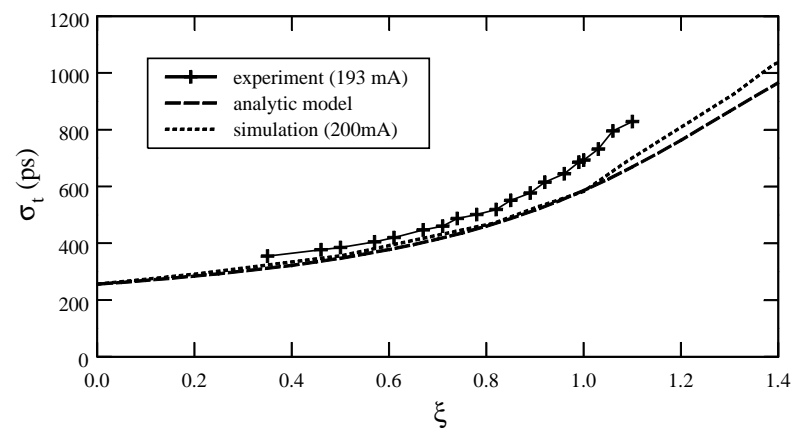

FIG. 10. Bunch length determined from experiment, analytic modeling, and simulations, for active Landau-cavity operation with the Aladdin lattice. The parameter $\xi$ is proportional to the Landau-cavity voltage, equaling unity for an optimally lengthened bunch.

measurement of bunch length is increased by $\sim 100$ ps by the time response of the capacitive pickup.

When $\xi$ was increased above 1.25 for $I=84$ or $193 \mathrm{~mA}$, a loss of beam current occurred. At this level of the Landau cavity voltage, the fast feedback amplifiers on the rf system are saturating so that the effective value of $\beta_{2}$ is decreased. Analytic modeling predicts that saturation of the feedback gain will result in Robinson instabilities if $\beta_{2}$ drops below $\sim 50$. Thus, a possible explanation for the beam losses is the onset of Robinson instabilities caused by saturation of the fast rf feedback.

A previous test of feedback where $\beta_{2}$ was estimated to equal 40 displayed unstable operation, consistent with analytic modeling and simulations. In normal Aladdin operations, the harmonic cavity is used actively with an estimated value of $\beta_{2}=160$, for currents of $0-300 \mathrm{~mA}$, achieving stable operation for optimally lengthened bunches.

Thus, for active operation of the Landau cavity with the Aladdin lattice, the analytic modeling, simulations, and experiments are in approximate agreement.

\section{ACTIVE LANDAU CAVITY WITH THE LF20 LATTICE}

\section{A. Analytic modeling}

In Fig. 11(a), uncoupled dipole and quadrupole Robinson instabilities are predicted for an optimally lengthened bunch, for active Landau-cavity operation with the LF20 lattice and a fundamental rf voltage of $50 \mathrm{kV}$. For large feedback gains where $\beta_{2}>170$, the equilibrium phase instability is predicted when the ring current exceeds $200 \mathrm{~mA}$.

When dipole-quadrupole mode coupling is included in the analysis [Fig. 11(b)], a fast mode-coupling instability is predicted for $\beta_{2}<130$, even though the dipole and quadrupole mode frequencies differ greatly in the 
uncoupled-mode analysis. The prevalence of equilibrium phase and fast mode-coupling instabilities indicates that frequency shifts produced by the beam current are comparable to the Robinson frequency $\omega_{R}$, whose value is small when using the LF20 lattice. Stable operation with a current of $300 \mathrm{~mA}$ is predicted for a narrow range of $\beta_{2}$ values with $130<\beta_{2}<170$. Nearly the same predictions for optimally lengthened bunches were obtained by
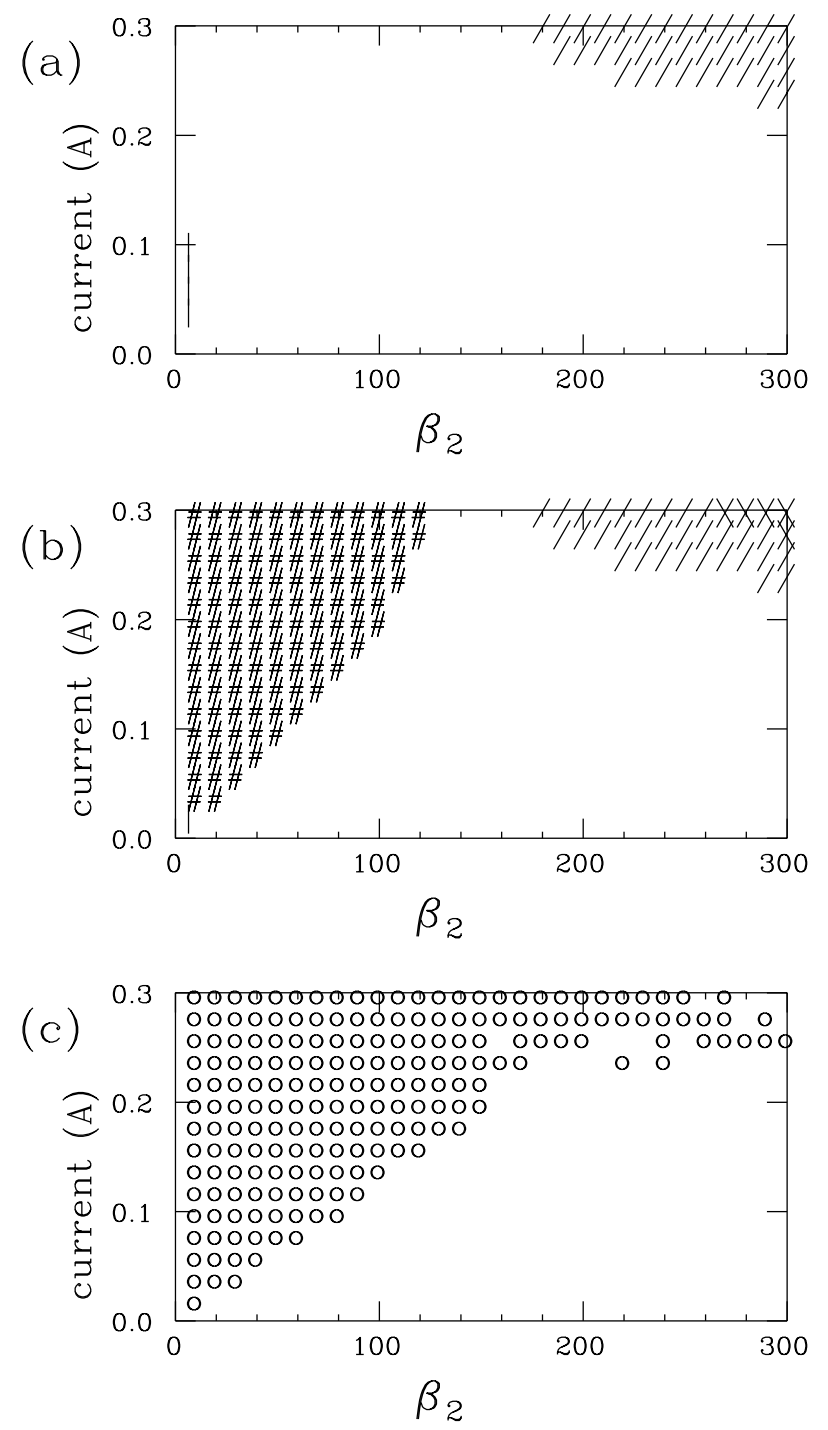

FIG. 11. (a) Robinson instabilities are predicted without consideration of mode coupling, for active Landau-cavity operation with optimally lengthened bunches in the LF20 lattice, with a fundamental rf voltage of $50 \mathrm{kV}$. A symbol is plotted when Robinson instability is predicted for a given ring current and effective rf coupling of the Landau-cavity $\left(\beta_{2}\right)$. Vertical line: dipole instability; /: equilibrium phase instability. (b) Dipole-quadrupole mode coupling is included in Robinson instability predictions. Vertical line: coupled-dipole instability; \#: fast mode-coupling instability; /: equilibrium phase instability; \: zero-frequency coupled dipole-quadrupole instability. (c) Instabilities observed in 500000 turn simulations. using the quartic-potential formula for the coupled dipolequadrupole instability [Eq. (B5)]. Simulation results (described below) are shown in Fig. 11(c).

In Fig. 12, we include dipole-quadrupole mode coupling to model bunches with $0<\xi<2$, for the cases where $\beta_{2}=40,80$, and 160. For the lower values of $\beta_{2}$, a fast mode-coupling instability is predicted when $\xi \sim 1$. The equilibrium phase instability is predicted for $\beta_{2}=$ 160 when a large current $(\sim 300 \mathrm{~mA})$ is lengthened with $\xi \approx 1$. A coupled-bunch instability with mode number of 1 is predicted for large currents and small values of $\xi$.

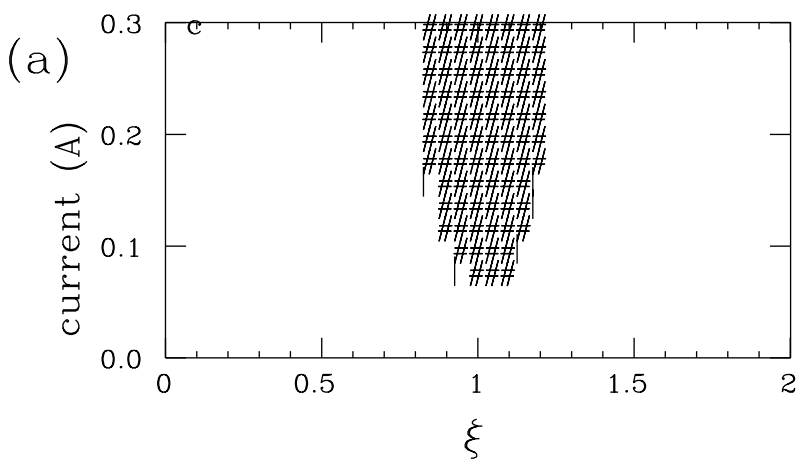

(b)
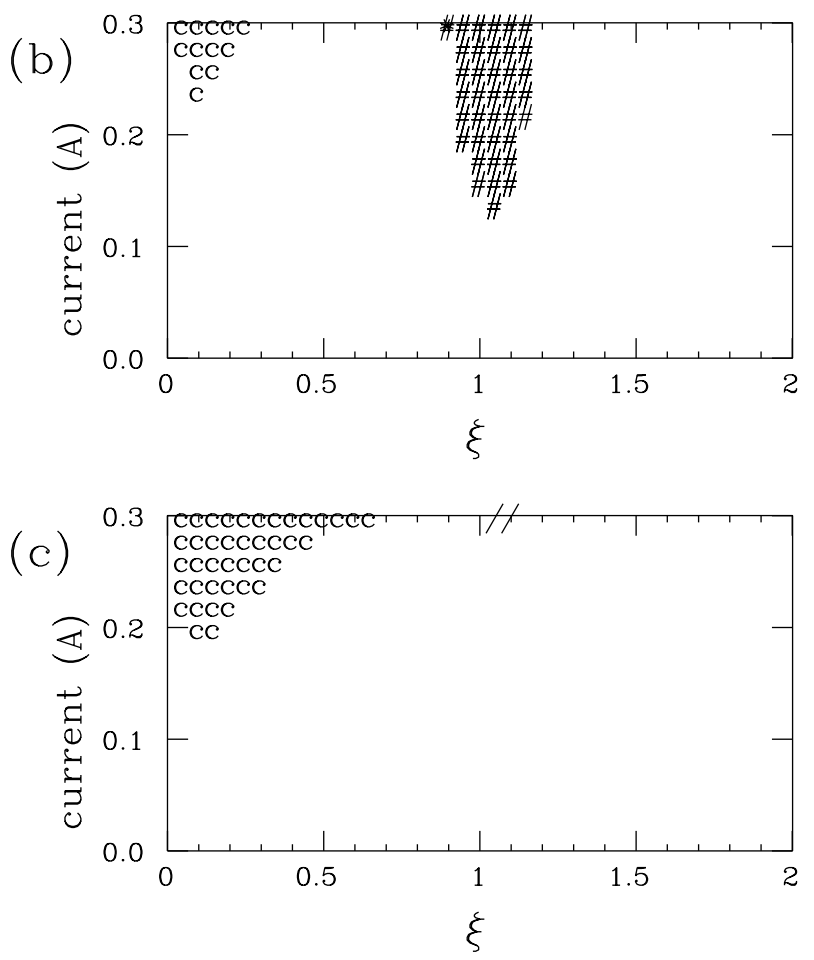

FIG. 12. Instability predictions which include dipolequadrupole mode coupling for active Landau-cavity operation with the LF20 lattice for three values of the Landau-cavity effective rf coupling $\left(\beta_{2}\right)$. Vertical line: coupled-dipole Robinson instability; *: coupled-quadrupole Robinson instability; \#: fast mode-coupling Robinson instability; /: equilibrium phase instability; c: coupled-bunch instability with longitudinal mode number of 1. (a) $\beta_{2}=40$, (b) $\beta_{2}=80$, and (c) $\beta_{2}=160$. 


\section{B. Simulations}

For optimally lengthened bunches, Fig. 11(c) shows instabilities observed in simulations, where a final energy spread exceeding the natural value by $10 \%$ is taken to indicate instability. The simulations are in approximate agreement with Fig. 11(b), in which dipole-quadrupole mode coupling is modeled. While the analytic modeling predicts a narrow range of $\beta_{2}$ values for which optimally lengthened $300 \mathrm{~mA}$ beams are stable, the simulations predict instability when $I \geq 260 \mathrm{~mA}$ for all values of $\beta_{2}$ considered.

In Fig. 13, simulations are shown for comparison with the analytic modeling of Fig. 12. The simulations display a region of Robinson instability for optimally lengthened
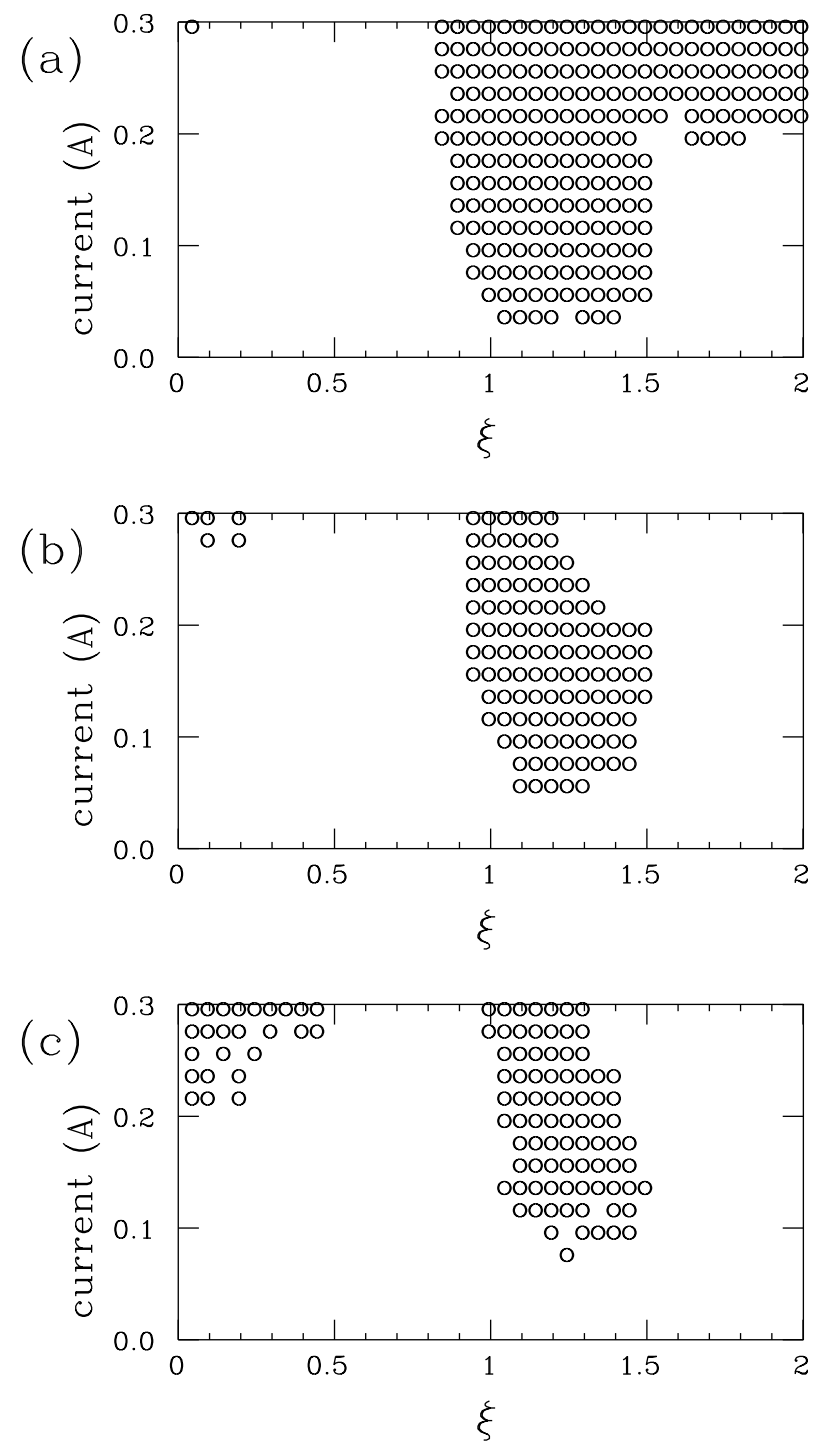

FIG. 13. Instabilities observed in 500000 turn simulations of active Landau-cavity operation with the LF20 lattice for three values of the active Landau-cavity effective rf coupling $\left(\beta_{2}\right)$. (a) $\beta_{2}=40$, (b) $\beta_{2}=80$, and (c) $\beta_{2}=160$. and double-hump bunches, consistent with the fast modecoupling instability predicted in Fig. 12. Optimally lengthened bunches are unstable for $I \geq 120 \mathrm{~mA}$ when $\beta_{2}=$ 80; they are unstable for $I \geq 260 \mathrm{~mA}$ when $\beta_{2} \geq 160$. Throughout most of the $0-300 \mathrm{~mA}$ current range, stable operation with optimally lengthened bunches is obtained, provided that $\beta_{2} \geq 160$.

For single-hump bunches $(\xi \leq 1)$, the simulations are in approximate agreement with the analytic modeling. However, simulations of double-hump bunches may display instability that is not predicted by the analytic model. Such a discrepancy may result if the collective oscillation frequency of double-hump bunches $\left(\omega_{R}\right)$ is overestimated by the rigid dipole model, if the frequency shift resulting from the beam current is underestimated in the analytic model, or if coupling to higher-order synchrotron modes (e.g., sextupole and octupole) becomes important in these lengthened bunches.

Figure 14 displays simulated unstable behavior for a current of $300 \mathrm{~mA}, \beta_{2}=160$, and $\xi=1.03$. According to analytic modeling, the system is at the threshold of equilibrium phase instability. The bunch position and length undergo relaxation oscillations of comparable magnitude, during which the relative energy spread oscillates between its natural value of $4.8 \times 10^{-4}$ and $\sim 1.5 \times 10^{-3}$.

With an increased fundamental rf voltage of $90 \mathrm{kV}$ (identical to that of standard Aladdin operation), the equilibrium phase instability is no longer predicted for LF20 with currents of $0-300 \mathrm{~mA}$. For $0-300 \mathrm{~mA}$, stable optimally lengthened bunches are predicted analytically for $\beta_{2} \geq 110$. Simulations for $\beta_{2}=160$, shown in Fig. 15, indicate that double-hump bunches with $\xi$ slightly greater than 1 are unstable for $I \geq 200 \mathrm{~mA}$. In simulations for $\beta_{2}=80$, optimally lengthened bunches are unstable when $I \geq 160 \mathrm{~mA}$. For $90 \mathrm{kV}$ rf operation of LF20, simulations of optimally lengthened bunches are stable for $0-300 \mathrm{~mA}$, provided that $\beta_{2} \geq 160$ [29].

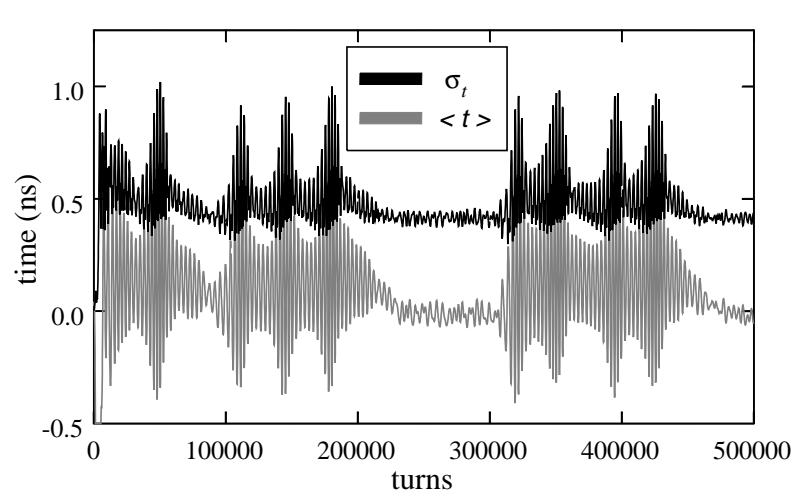

FIG. 14. Simulation of Robinson instability for active Landaucavity operation with the LF20 lattice, for a ring current of $300 \mathrm{~mA}, \beta_{2}=160$, and $\xi=1.03$. 


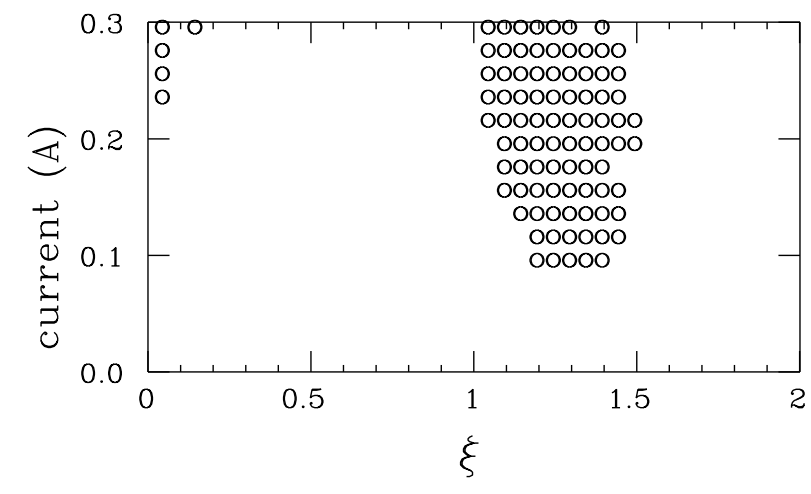

FIG. 15. Instabilities observed in 500000 turn simulations of active Landau-cavity operation with the LF20 lattice and $\beta_{2}=$ 160 , with an increased fundamental rf voltage of $90 \mathrm{kV}$.

\section{Experiment}

Experimental studies of active harmonic cavity operation with the LF20 lattice and $V_{T 1}=50 \mathrm{kV}$ were performed with an estimated value of $\beta_{2}=160$. For currents of 100-200 mA, coupled-bunch instabilities were suppressed when the Landau cavity voltage was sufficiently large that $\xi$ exceeded $0.6-0.85$, in rough agreement with the analytic estimate that $\xi$ must exceed $0.8-0.9$ to suppress coupled-bunch instability.

For a ring current of $16.5 \mathrm{~mA}$, an apparent Robinson instability was observed when $\xi>1.14$. For a ring current of $104 \mathrm{~mA}$, apparent Robinson instabilities were observed when $0.81<\xi<1.24$, and when $\xi>1.41$. For ring currents of 160 and $174 \mathrm{~mA}$, instability was observed for $\xi>0.84$ and $\xi>0.74$, respectively. For these higher currents, beam losses occurred when $\xi$ was increased above the threshold values.

The experimental results approximately agree with the modeling and simulations of Figs. 12 and 13. The modeling and simulations for $\beta_{2}$ equaling 40 or 80 agree closely with experiment, suggesting that the value of $\beta_{2}$ in the experiment is less than 160. This could result if the feedback gain is less than the low-signal value used to estimate $\beta_{2}$. In addition, there is considerable uncertainty in the experimental values of $\alpha, \sigma_{E} / E$, and $\xi$.

In Fig. 16(a), we display the bunch oscillation frequency observed on a spectrum analyzer connected to a beam phase detector circuit for an extremely low current of $1.5 \mathrm{~mA}$. The observed frequency is $\sim 20 \%$ higher than the analytically calculated coupled-dipole frequency, which nearly equals $\omega_{R} / 2 \pi$ for this low ring current. This discrepancy may arise from the experimental momentum compaction exceeding the value used in the calculation. With a current of $155 \mathrm{~mA}$, two oscillation frequencies are observed with the values shown in Fig. 16(b). When the frequencies converge for $\xi=0.89$, instability results in which the oscillation amplitude increases by $18 \mathrm{~dB}$ (a factor of 8). Also shown are analytic calculations of the
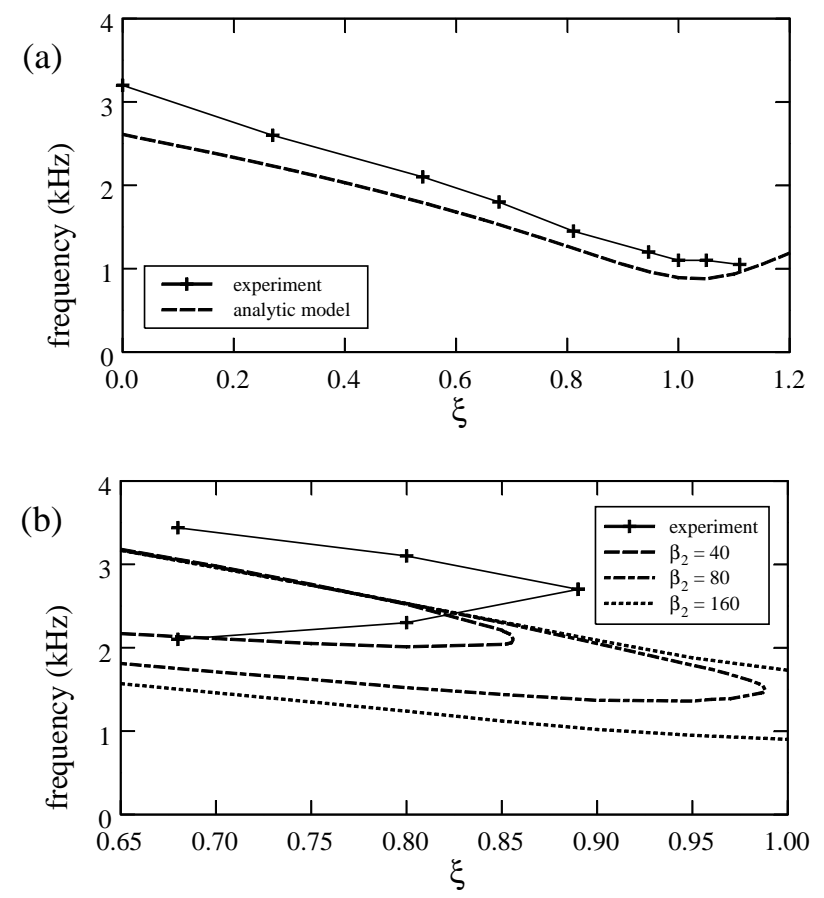

FIG. 16. (a) Observed bunch oscillation frequency as a function of $\xi$, for active Landau-cavity operation with the LF20 lattice and a ring current of $1.5 \mathrm{~mA}$. Analytic modeling of the coupled-dipole Robinson frequency is also shown. (b) Bunch oscillation frequencies observed for active Landau-cavity operation with the LF20 lattice at a ring current of $155 \mathrm{~mA}$. Instability occurs when the frequencies converge for $\xi=0.89$. Analytic results for the coupled-dipole and coupled-quadrupole mode frequencies are shown for $\beta_{2}=40,80$, and 160 .

coupled-dipole and coupled-quadrupole mode frequencies for $\beta_{2}$ equaling 40,80 , and 160 . Comparison with the analytic calculations suggests that the value of $\beta_{2}$ in the experiment is less than 160 . This data supports our interpretation of instability resulting from dipole-quadrupole mode coupling.

For active harmonic cavity operation with single-hump bunches in the LF20 lattice, the analytic modeling of dipole-quadrupole mode coupling, simulations, and experiment are in approximate agreement. For double-hump bunches, instabilities are more prevalent in simulations than in the analytic model.

\section{DISCUSSION}

Using analytic modeling, simulations, and experiments, we have studied Robinson instabilities when a Landau cavity is utilized with the standard Aladdin lattice and the low-emittance LF20 lattice, whose momentum compaction is much smaller. Passive and active operation of the Landau cavity were considered. In our analytic model, we estimate the frequency of collective dipole oscillations by approximating the dipole motion as rigid. Instabilities are modeled by substituting the estimated collective frequency 
for the synchrotron frequency in formulas applicable to a quadratic synchrotron potential. This model is expected to predict dipole and quadrupole mode frequencies and growth rates within $\sim 10 \%$ for single-hump bunches.

For single-hump bunches and passive operation with double-hump bunches, analytic modeling, simulations, and experiments are in approximate agreement, provided that dipole-quadrupole mode coupling is included in the analytic modeling. For active operation with double-hump bunches, instabilities are more prevalent in simulations than in the analytic model.

With the Aladdin lattice and a $90 \mathrm{kV}$ rf voltage, passive operation of the Landau cavity gives stable optimally lengthened bunches for currents of $120-300 \mathrm{~mA}$, while active operation succeeds for currents of $0-300 \mathrm{~mA}$. Landau-cavity operation with the LF20 lattice and a $50 \mathrm{kV}$ rf voltage suffers from increased instability resulting from coupling of the dipole and quadrupole Robinson modes. Studies of LF20 with a $90 \mathrm{kV}$ rf voltage confirm that the increased instability results from the small momentum compaction of the LF20 lattice rather than the lower rf voltage [29].

The mode-coupling Robinson instabilities limit the utility of the Landau cavity for active operation with the LF20 lattice. According to analytic modeling and simulations, several options exist for improving this situation. Increasing the effective rf-coupling coefficient of the Landau cavity is predicted to increase stability; this may be accomplished by a modification of the feedback circuitry and/or rf cavity. Modeling and simulations show slightly better stability with the higher rf voltages we intend to use if the momentum aperture of the LF20 lattice is successfully increased. Stability may also be improved by using a lattice which has a larger momentum compaction, in which case the emittance may also be increased, since emittance is roughly proportional to momentum compaction. In addition, our analytic modeling and simulation techniques may be used to find rf-cavity parameters that are more conducive to stability.

\section{ACKNOWLEDGMENTS}

The authors thank W. S. Trzeciak, D. E. Eisert, and the Aladdin operations group for experimental assistance, M. A. Green, N. Towne, J. M. Byrd, and K. C. Harkay for helpful discussions, and E. Weihreter and C.S. Hsue for suggesting this research topic. This work was supported by NSF Grant No. DMR-0084402.

\section{APPENDIX A: SINGLE-MODE ROBINSON INSTABILITIES IN A QUARTIC SYNCHROTRON POTENTIAL}

According to Eq. (9.11) of Ref. [15], coherent Robinson oscillations in a quartic synchrotron potential obey $\rho_{m}=$ $\sum_{n} T_{m, n} \rho_{n}$, where $m$ and $n$ take on the values $M j(j=$ $-\infty, \ldots, \infty)$ and $M$ is the number of rf buckets. For $\left|\alpha_{R}\right| \ll$ $|\Omega|, T_{m, n}$ may be written in our notation as

$$
T_{m, n}=-i n Z_{n}\left[\frac{\alpha e \omega_{\mathrm{o}}^{2} C_{5} I}{2 \pi E \Delta \omega_{s}^{2}}\right] \frac{4}{\left(n r_{\mathrm{o}}\right)^{2}} \sum_{\mu=1}^{\infty} \int_{0}^{\infty} d x \frac{x^{4} e^{-x^{4}} J_{\mu}\left(n r_{\mathrm{o}} x\right) J_{\mu}\left(m r_{\mathrm{o}} x\right)}{x^{2}-\left(\Omega-i \alpha_{R}\right)^{2} /\left(\mu \Delta \omega_{s}\right)^{2}},
$$

where $C_{5}=4.686, r_{\mathrm{o}}=1.720 \omega_{\mathrm{o}} \sigma_{t}$, and $Z_{n} \equiv Z\left(n \omega_{\mathrm{o}}+\Omega\right)$. Here, $\mu$ denotes the mode number, equaling 1 for a dipole mode, 2 for a quadrupole mode, etc. The synchrotron frequency spread is parametrized by $\Delta \omega_{s}^{2}=\left(\alpha e \omega_{g}^{3} / 2.825 E T_{\mathrm{o}}\right) \times$ $\left(\nu^{2}-1\right) \sigma_{t}^{2} V_{T 1} \sin \psi_{1}$. The rms synchrotron frequency spread obeys $\sigma_{\omega_{s}}=\Delta \omega_{s} / 1.720$. A comparison with Eq. (11) indicates that the rigid dipole model predicts a Robinson frequency of $\omega_{R}=1.19 \Delta \omega_{s}$.

When mode coupling is negligible, there are oscillations for which only one value of $\mu$, denoted $\mu_{\mathrm{o}}$, contributes to $T_{m, n}$. In this case, $T_{m, n} \approx T_{m, n}^{\left(\mu_{\mathrm{o}}\right)}$, where

$$
T_{m, n}^{\left(\mu_{\mathrm{o}}\right)}=-i n Z_{n}\left[\frac{\alpha e \omega_{\mathrm{o}}^{2} C_{5} I}{2 \pi E \Delta \omega_{s}^{2}}\right] \frac{4}{\left(n r_{\mathrm{o}}\right)^{2}} \int_{0}^{\infty} d x \frac{x^{4} e^{-x^{4}} J_{\mu_{\mathrm{o}}}\left(n r_{\mathrm{o}} x\right) J_{\mu_{\mathrm{o}}}\left(m r_{\mathrm{o}} x\right)}{x^{2}-\left(\Omega-i \alpha_{R}\right)^{2} /\left(\mu_{\mathrm{o}} \Delta \omega_{s}\right)^{2}}
$$

Just above the threshold of instability, $\alpha_{R}=0^{-}$; in this case the Plemelj formula [30] gives

$$
\begin{aligned}
T_{m, n}^{\left(\mu_{\mathrm{o}}\right)}=-i n Z_{n}\left[\frac{\alpha e \omega_{\mathrm{o}}^{2} C_{5} I}{2 \pi E \Delta \omega_{s}^{2}}\right] \frac{4}{\left(n r_{\mathrm{o}}\right)^{2}}[ & P \int_{0}^{\infty} d x \frac{x^{4} \exp \left(-x^{4}\right) J_{\mu_{\mathrm{o}}}\left(n r_{\mathrm{o}} x\right) J_{\mu_{\mathrm{o}}}\left(m r_{\mathrm{o}} x\right)}{x^{2}-\Omega^{2} /\left(\mu_{\mathrm{o}} \Delta \omega_{s}\right)^{2}} \\
& \left.+\left.\frac{i \pi x_{\mu_{\mathrm{o}}}^{4} \exp \left(-x_{\mu_{\mathrm{o}}}^{4}\right) J_{\mu_{\mathrm{o}}}\left(n r_{\mathrm{o}} x_{\mu_{\mathrm{o}}}\right) J_{\mu_{\mathrm{o}}}\left(m r_{\mathrm{o}} x_{\mu_{\mathrm{o}}}\right)}{2 \Omega /\left(\mu_{\mathrm{o}} \Delta \omega_{s}\right)}\right|_{x_{\mu_{\mathrm{o}}}=\Omega / \mu_{\mathrm{o}} \Delta \omega_{s}}\right] .
\end{aligned}
$$


For a sufficiently short bunch length, we utilize the fact that $J_{\mu}(x) \propto x^{\mu}$ for small $x$ to obtain

$$
\begin{aligned}
& T_{m, n}^{\left(\mu_{\mathrm{o}}\right)}=-i n Z_{n}\left[\frac{\alpha e \omega_{\mathrm{o}}^{2} C_{5} I}{2 \pi E \Delta \omega_{s}^{2}}\right] \frac{4}{\left(n r_{\mathrm{o}}\right)^{2}} \\
& \times\left[P \int_{0}^{\infty} d x \frac{x^{4+2 \mu_{\mathrm{o}}} \exp \left(-x^{4}\right)\left[x^{-\mu_{\mathrm{o}}} J_{\mu_{\mathrm{o}}}\left(n r_{\mathrm{o}} x\right)\right]\left[x^{-\mu_{\mathrm{o}}} J_{\mu_{\mathrm{o}}}\left(m r_{\mathrm{o}} x\right)\right]}{x^{2}-\Omega^{2} /\left(\mu_{\mathrm{o}} \Delta \omega_{s}\right)^{2}}\right. \\
& \left.+\left.\frac{i \pi x_{\mu_{\mathrm{o}}}^{4} \exp \left(-x_{\mu_{\mathrm{o}}}^{4}\right) J_{\mu_{\mathrm{o}}}\left(n r_{\mathrm{o}} x_{\mu_{\mathrm{o}}}\right) J_{\mu_{\mathrm{o}}}\left(m r_{\mathrm{o}} x_{\mu_{\mathrm{o}}}\right)}{2 \Omega /\left(\mu_{\mathrm{o}} \Delta \omega_{s}\right)}\right|_{x_{\mu_{\mathrm{o}}}=\Omega / \mu_{\mathrm{o}} \Delta \omega_{s}}\right] \\
& \approx-i n Z_{n}\left[\frac{\alpha e \omega_{\mathrm{o}}^{2} C_{5} I}{2 \pi E \Delta \omega_{s}^{2}}\right] \frac{4}{\left(n r_{\mathrm{o}}\right)^{2}} x_{\mu_{\mathrm{o}}}^{-2 \mu_{\mathrm{o}}} J_{\mu_{\mathrm{o}}}\left(n r_{\mathrm{o}} x_{\mu_{\mathrm{o}}}\right) J_{\mu_{\mathrm{o}}}\left(m r_{\mathrm{o}} x_{\mu_{\mathrm{o}}}\right) \\
& \times\left.\left[P \int_{0}^{\infty} d x \frac{x^{4+2 \mu_{0}} \exp \left(-x^{4}\right)}{x^{2}-\Omega^{2} /\left(\mu_{\mathrm{o}} \Delta \omega_{s}\right)^{2}}+\frac{i \pi x_{\mu_{\mathrm{o}}}^{4+2 \mu_{\mathrm{o}}} \exp \left(-x_{\mu_{\mathrm{o}}}^{4}\right)}{2 \Omega /\left(\mu_{\mathrm{o}} \Delta \omega_{s}\right)}\right]\right|_{x_{\mu_{0}}=\Omega / \mu_{\mathrm{o}} \Delta \omega_{s}}
\end{aligned}
$$

Note that $T_{m, n}^{\left(\mu_{0}\right)}$ may be written as $A_{m}^{\left(\mu_{\mathrm{o}}\right)} B_{n}^{\left(\mu_{\mathrm{o}}\right)}$ (i.e., $T_{m, n}^{\left(\mu_{\mathrm{o}}\right)}$ is of rank $\leq 1$ [15]) so that $\rho_{m}=\sum_{n} T_{m, n} \rho_{n}$ has solution when $\sum_{n} T_{n, n}^{\left(\mu_{\mathrm{o}}\right)}=1$.

We now consider a real impedance $Z$. In the case of a quadratic synchrotron potential, such an impedance causes growth or damping without shifting the real Robinson frequency. In order that $\sum_{n} T_{n, n}^{\left(\mu_{0}\right)}=1$, we must have $\operatorname{Im}\left(\sum_{n} T_{n, n}^{\left(\mu_{0}\right)}\right)=0$, which is satisfied when the real oscillation frequency $\Omega$ obeys

$$
P \int_{0}^{\infty} d x \frac{x^{4+2 \mu_{\mathrm{o}}} \exp \left(-x^{4}\right)}{x^{2}-\Omega^{2} /\left(\mu_{\mathrm{o}} \Delta \omega_{s}\right)^{2}}=P \int_{0}^{\infty} d x \frac{x^{4+2 \mu_{\mathrm{o}}} \exp \left(-x^{4}\right)}{x^{2}-x_{\mu_{\mathrm{o}}}^{2}}=0 .
$$

Evaluating Eq. (A5) yields $x_{1}=1.076, x_{2}=1.164, x_{3}=1.236, x_{4}=1.298$, etc. Thus, at the threshold of dipole Robinson instability, the oscillation frequency obeys $\Omega=1.076 \Delta \omega_{s}=0.90 \omega_{R}$, where $\omega_{R}\left(=1.19 \Delta \omega_{s}\right)$ is the dipole Robinson frequency calculated in Eq. (11) by assuming a rigid-bunch oscillation. For the quadrupole mode $\left(\mu_{\mathrm{o}}=2\right)$, $\Omega=1.164\left(2 \Delta \omega_{s}\right)=0.98\left(2 \omega_{R}\right)$. For the sextupole mode, $\Omega=1.236\left(3 \Delta \omega_{s}\right)=1.04\left(3 \omega_{R}\right)$, while, for the octupole mode, $\Omega=1.298\left(4 \Delta \omega_{s}\right)=1.09\left(4 \omega_{R}\right)$. For the dipole, quadrupole, sextupole, and octupole modes, the Robinson oscillation frequencies at threshold in a quartic potential are within $10 \%$ of the value $\mu_{\mathrm{o}} \omega_{R}$, where $\omega_{R}$ is the dipole oscillation frequency obtained in Sec. II by assuming a rigid dipole oscillation.

The beam current $I$ at the instability threshold for the $\mu_{\mathrm{o}}$ th mode follows from $\operatorname{Re}\left(\sum_{n} T_{n, n}^{\left(\mu_{\mathrm{o}}\right)}\right)=1$ :

$$
1=\sum_{n} n Z_{n}\left[\frac{\alpha e \omega_{\mathrm{o}}^{2} C_{5} I}{2 \pi E \Delta \omega_{s}^{2}}\right] \frac{4}{\left(n r_{\mathrm{o}}\right)^{2}}\left[\left.\frac{\pi x_{\mu_{\mathrm{o}}}^{4} \exp \left(-x_{\mu_{\mathrm{o}}}^{4}\right) J_{\mu_{\mathrm{o}}}^{2}\left(n r_{\mathrm{o}} x_{\mu_{\mathrm{o}}}\right)}{2 x_{\mu_{\mathrm{o}}}}\right|_{x_{\mu_{0}}=\Omega / \mu_{\mathrm{o}} \Delta \omega_{s}}\right] .
$$

Far above the instability threshold, we have

$$
\begin{aligned}
T_{m, n}^{\left(\mu_{\mathrm{o}}\right)} & =-i n Z_{n}\left[\frac{\alpha e \omega_{\mathrm{o}}^{2} C_{5} I}{2 \pi E \Delta \omega_{s}^{2}}\right] \frac{4}{\left(n r_{\mathrm{o}}\right)^{2}}\left[\int_{0}^{\infty} d x \frac{x^{4+2 \mu_{\mathrm{o}}} \exp \left(-x^{4}\right)\left[x^{-\mu_{\mathrm{o}}} J_{\mu_{\mathrm{o}}}\left(n r_{\mathrm{o}} x\right)\right]\left[x^{-\mu_{\mathrm{o}}} J_{\mu_{\mathrm{o}}}\left(m r_{\mathrm{o}} x\right)\right]}{x^{2}-\left(\Omega-i \alpha_{R}\right)^{2} /\left(\mu_{\mathrm{o}} \Delta \omega_{s}\right)^{2}}\right] \\
& \approx-i n Z_{n}\left[\frac{\alpha e \omega_{\mathrm{o}}^{2} C_{5} I}{2 \pi E \Delta \omega_{s}^{2}}\right] \frac{4}{\left(n r_{\mathrm{o}}\right)^{2}} \frac{x \mu_{\mathrm{o}}^{-2 \mu_{\mathrm{o}}} J_{\mu_{\mathrm{o}}}\left(n r_{\mathrm{o}} x_{\mu_{\mathrm{o}}}\right) J_{\mu_{\mathrm{o}}}\left(m r_{\mathrm{o}} x_{\mu_{\mathrm{o}}}\right)}{x_{\mu_{\mathrm{o}}}^{2}-\left(\Omega-i \alpha_{R}\right)^{2} /\left(\mu_{\mathrm{o}} \Delta \omega_{s}\right)^{2}}\left[\int_{0}^{\infty} d x x^{4+2 \mu_{\mathrm{o}}} \exp \left(-x^{4}\right)\right]
\end{aligned}
$$

In the absence of mode coupling, $T_{m, n}=T_{m, n}^{\left(\mu_{0}\right)}$, where $T_{m, n}^{\left(\mu_{0}\right)}$ is of rank $\leq 1$, so that instability requires $\sum_{n} T_{n, n}^{\left(\mu_{0}\right)}=1$. According to Eq. (A7), this gives

$$
\begin{aligned}
\left(\Omega-i \alpha_{R}\right)^{2} & =\left(x_{\mu_{\mathrm{o}}} \mu_{\mathrm{o}} \Delta \omega_{s}\right)^{2}+\mu_{\mathrm{o}}^{2} \sum_{n} i n Z_{n}\left[\frac{\alpha e \omega_{\mathrm{o}}^{2} C_{5} I}{2 \pi E}\right] \frac{4}{\left(n r_{\mathrm{o}}\right)^{2}} x_{\mu_{\mathrm{o}}}^{-2 \mu_{\mathrm{o}}} J_{\mu_{\mathrm{o}}}^{2}\left(n r_{\mathrm{o}} x_{\mu_{\mathrm{o}}}\right) \int_{0}^{\infty} d x x^{4+2 \mu_{\mathrm{o}}} \exp \left(-x^{4}\right) \\
& \equiv\left(x_{\mu_{\mathrm{o}}} \mu_{\mathrm{o}} \Delta \omega_{s}\right)^{2}+i \delta \Omega_{\mu_{\mathrm{o}}}^{2} .
\end{aligned}
$$

For a real impedance, our assumption that the instability threshold is exceeded requires that the RHS of Eq. (A6) exceed 1. Because both the real and the imaginary components of a complex frequency shift are effective in overcoming Landau damping [15,23], we generalize this criterion for an arbitrary impedance by requiring that the RHS of Eq. (A6) have 
magnitude exceeding 1 , which is equivalent to

$$
\left|\delta \Omega_{\mu_{\mathrm{o}}}^{2}\right|>\left(\mu_{\mathrm{o}} \Delta \omega_{s}\right)^{2}\left[\frac{2 \int_{0}^{\infty} d x x^{4+2 \mu_{\mathrm{o}}} \exp \left(-x^{4}\right)}{\pi x_{\mu_{\mathrm{o}}}^{2 \mu_{\mathrm{o}}+3} \exp \left(-x_{\mu_{\mathrm{o}}}^{4}\right)}\right] .
$$

For the dipole mode, this criterion for overcoming Landau damping becomes

$$
\left|\delta \Omega_{1}^{2}\right|^{1 / 2}>0.622 \Delta \omega_{s} .
$$

Equation (A10) was obtained in a different manner in Eq. (9.28) of Ref. [15]. For the quadrupole, sextupole, or octupole modes, Landau damping is overcome, according to Eq. (A9), when

$$
\left|\delta \Omega_{2}^{2}\right|^{1 / 2}>0.625\left(2 \Delta \omega_{s}\right), \quad\left|\delta \Omega_{3}^{2}\right|^{1 / 2}>0.626\left(3 \Delta \omega_{s}\right), \quad\left|\delta \Omega_{4}^{2}\right|^{1 / 2}>0.627\left(4 \Delta \omega_{s}\right) .
$$

For the dipole Robinson mode, we expand the Bessel function in Eq. (A8) using

$$
J_{\mu_{\mathrm{o}}}\left(n r_{\mathrm{o}} x_{\mu_{\mathrm{o}}}\right) \approx \frac{1}{\Gamma\left(\mu_{\mathrm{o}}+1\right)}\left(\frac{n r_{\mathrm{o}} x_{\mu_{\mathrm{o}}}}{2}\right)^{\mu_{\mathrm{o}}}\left[1-\frac{1}{\mu_{\mathrm{o}}+1}\left(\frac{n r_{\mathrm{o}} x_{\mu_{\mathrm{o}}}}{2}\right)^{2}\right]
$$

to obtain

$$
\left(\Omega-i \alpha_{R}\right)^{2}=\left(1.076 \Delta \omega_{s}\right)^{2}+\frac{i \alpha e \omega_{0}^{2} I}{2 \pi E}(1.076) \sum_{n} n Z_{n} F_{0.93 n \omega_{0}}^{2},
$$

where, to order $\sigma_{t}^{2}, F_{0.93 n \omega_{0}}$ is the bunch form factor evaluated at angular frequency $0.93 n \omega_{0}$. In contrast, the assumption of a rigid dipole oscillation gives the $\mu_{\mathrm{o}}=1$ term of Eq. (12),

$$
\left(\Omega-i \alpha_{R}\right)^{2}=\omega_{R}^{2}+\frac{i \alpha e \omega_{\mathrm{o}}^{2} I}{2 \pi E} \sum_{n} n Z_{n} F_{n \omega_{\mathrm{o}}}^{2} .
$$

The low-current dipole Robinson frequency in a quartic potential, $1.076 \Delta \omega_{s}$, is given within $10 \%$ by the rigid-dipole value of $\omega_{R}=1.19 \Delta \omega_{s}$, while the coefficient of the coherent frequency shift term differs by $8 \%$, in addition to a $7 \%$ change in the frequencies where the form factors are evaluated. This confirms that Eq. (12) provides a good approximation for the dipole mode (frequency and growth rate given within $\sim 10 \%$ ) when the synchrotron potential is quartic.

For $\Omega>0$, let us define the coherent frequency shift $\Delta \Omega \equiv \Omega-i \alpha_{R}-1.076 \Delta \omega_{s}$ as the change in the complex Robinson frequency resulting from the beam current. The criterion for overcoming Landau damping given by Eq. (A10) may then be written as

$$
|\Delta \Omega|=\left|\frac{\delta \Omega_{1}^{2}}{2\left(1.076 \Delta \omega_{s}\right)}\right|>0.18 \Delta \omega_{s}=0.31 \sigma_{\omega_{s}} .
$$

For the quadrupole mode, expanding the Bessel function in Eq. (A8) yields

$$
\left(\Omega-i \alpha_{R}\right)^{2}=\left(1.164 \cdot 2 \Delta \omega_{s}\right)^{2}+\frac{i \alpha e \omega_{\mathrm{o}}^{2} I}{2 \pi E}(0.982) \sum_{n} n Z_{n}\left(n \omega_{\mathrm{o}} \sigma_{t}\right)^{2} F_{\sqrt{2 / 3} n \omega_{\mathrm{o}}} .
$$

In contrast, the $\mu_{\mathrm{o}}=2$ term of Eq. (12) gives

$$
\left(\Omega-i \alpha_{R}\right)^{2}=\left(2 \omega_{R}\right)^{2}+\frac{i \alpha e \omega_{\mathrm{o}}^{2} I}{2 \pi E} \sum_{n} n Z_{n}\left(n \omega_{\mathrm{o}} \sigma_{t}\right)^{2} F_{n \omega_{\mathrm{o}}}^{2} .
$$

The low-current quadrupole Robinson frequency in a quartic potential, $1.164\left(2 \Delta \omega_{s}\right)$, is given within $2 \%$ by Eq. (12), while the coherent frequency shift coefficient differs by $2 \%$, in addition to an $18 \%$ change in the frequencies where the form factors are evaluated. This confirms that Eq. (12) provides a good approximation for the quadrupole mode when the synchrotron potential is quartic.

Letting $\Delta \Omega$ denote the change in the complex quadrupole Robinson frequency resulting from the beam current $(\Delta \Omega \equiv$ $\left.\Omega-i \alpha_{R}-1.164 \cdot 2 \Delta \omega_{s}\right)$, the criterion for overcoming Landau damping given by Eq. (A11) may be written as

$$
|\Delta \Omega|=\left|\frac{\delta \Omega_{2}^{2}}{2\left(1.164 \cdot 2 \Delta \omega_{s}\right)}\right|>0.34 \Delta \omega_{s}=0.58 \sigma_{\omega_{s}} .
$$

For the sextupole mode, expanding the Bessel function in Eq. (A8) yields

$$
\left(\Omega-i \alpha_{R}\right)^{2}=\left(1.236 \cdot 3 \Delta \omega_{s}\right)^{2}+\frac{i \alpha e \omega_{\mathrm{o}}^{2} I}{2 \pi E}(0.258) \sum_{n} n Z_{n}\left(n \omega_{\mathrm{o}} \sigma_{t}\right)^{4} F_{0.75 n \omega_{\mathrm{o}}}^{2}
$$


In contrast, the $\mu_{\mathrm{o}}=3$ term of Eq. (12) gives

$$
\left(\Omega-i \alpha_{R}\right)^{2}=\left(3 \omega_{R}\right)^{2}+\frac{i \alpha e \omega_{0}^{2} I}{2 \pi E}(0.375) \sum_{n} n Z_{n}\left(n \omega_{0} \sigma_{t}\right)^{4} F_{n \omega_{0}}^{2} .
$$

The low-current sextupole Robinson frequency in a quartic potential, $1.236\left(3 \Delta \omega_{s}\right)$, is given within $4 \%$ by Eq. (12), while the coherent frequency shift coefficient differs by $31 \%$, in addition to a $25 \%$ change in the frequencies where the form factors are evaluated. This indicates that Eq. (12) overestimates the real and imaginary frequency shifts of the sextupole mode when the synchrotron potential is quartic.

Letting $\Delta \Omega$ denote the change in the complex sextupole Robinson frequency resulting from the beam current, the criterion for overcoming Landau damping given by Eq. (A11) may be written as

$$
|\Delta \Omega|=\left|\frac{\delta \Omega_{3}^{2}}{2\left(1.236 \cdot 3 \Delta \omega_{s}\right)}\right|>0.48 \Delta \omega_{s}=0.82 \sigma_{\omega_{s}} .
$$

For the octupole mode, expanding the Bessel function in Eq. (A8) yields

$$
\left(\Omega-i \alpha_{R}\right)^{2}=\left(1.298 \cdot 4 \Delta \omega_{s}\right)^{2}+\frac{i \alpha e \omega_{0}^{2} I}{2 \pi E}(0.0335) \sum_{n} n Z_{n}\left(n \omega_{\mathrm{o}} \sigma_{t}\right)^{6} F_{0.71 n \omega_{\mathrm{o}}}^{2}
$$

In contrast, the $\mu_{\mathrm{o}}=4$ term of Eq. (12) gives

$$
\left(\Omega-i \alpha_{R}\right)^{2}=\left(4 \omega_{R}\right)^{2}+\frac{i \alpha e \omega_{\mathrm{o}}^{2} I}{2 \pi E}(0.0833) \sum_{n} n Z_{n}\left(n \omega_{\mathrm{o}} \sigma_{t}\right)^{6} F_{n \omega_{\mathrm{o}}}^{2} .
$$

The low-current octupole Robinson frequency in a quartic potential, $1.298\left(4 \Delta \omega_{s}\right)$, is given within $9 \%$ by Eq. (12), while the coherent frequency shift coefficient differs by $60 \%$, in addition to a $29 \%$ change in the frequencies where the form factors are evaluated. This indicates that Eq. (12) substantially overestimates the real and imaginary frequency shifts for the octupole mode when the synchrotron potential is quartic.

Letting $\Delta \Omega$ denote the change in the complex octupole Robinson frequency resulting from the beam current, the criterion for overcoming Landau damping given by Eq. (A11) may be written as

$$
|\Delta \Omega|=\left|\frac{\delta \Omega_{4}^{2}}{2\left(1.298 \cdot 4 \Delta \omega_{s}\right)}\right|>0.61 \Delta \omega_{s}=1.04 \sigma_{\omega_{s}} .
$$

In summary, a single-mode analysis of the Robinson instability in a quartic potential indicates that Eq. (12) provides a good approximation for the dipole and quadrupole modes, while overestimating the coherent frequency shift for the sextupole and octupole modes.

\section{APPENDIX B: DIPOLE-QUADRUPOLE MODE COUPLING}

Let us include mode coupling between dipole and quadrupole Robinson modes by retaining the $\mu=1$ (dipole) and $\mu=2$ (quadrupole) terms of $T_{m, n}=\sum_{\mu=1}^{\infty} T_{m, n}^{(\mu)}$. For a quadratic synchrotron potential, Robinson oscillations with $\left|\alpha_{R}\right| \ll|\Omega|$ obey [15,22]

$$
T_{m, n}^{(\mu)}=-i \frac{Z_{n}}{n}\left[\frac{\alpha e \omega_{\mathrm{o}}^{2} I}{2 \pi E \omega_{s}^{2}}\right] \frac{F_{m \omega_{0}} F_{n \omega_{\mathrm{o}}}}{\mu ! 2^{\mu-1}} \frac{m^{\mu} n^{\mu}\left(\omega_{\mathrm{o}} \sigma_{t}\right)^{2 \mu-2}}{1-\left(\Omega-i \alpha_{R}\right)^{2} /\left(\mu \omega_{s}\right)^{2}} .
$$

Thus, we have

$$
T_{m, n}^{(\mu)}=(m / n)^{\mu}\left(F_{m \omega_{0}} / F_{n \omega_{0}}\right) T_{n, n}^{(\mu)} .
$$

For $T_{m, n}=T_{m, n}^{(1)}+T_{m, n}^{(2)}$, Eq. (B2) implies that $\rho_{m}=\sum_{n} T_{m, n} \rho_{n}$ has a nontrivial solution when

$$
\left(\sum_{n} T_{n, n}^{(1)}-1\right)\left(\sum_{n} T_{n, n}^{(2)}-1\right)=\left(\sum_{n} n T_{n, n}^{(1)}\right)\left(\sum_{n}(1 / n) T_{n, n}^{(2)}\right) .
$$

When the RHS (which is $\propto I^{2}$ ) is negligible, Eq. (B3) reduces to the dipole and quadrupole single-mode formulas. To include radiation damping, we make the substitution $\alpha_{R} \rightarrow \alpha_{R}-\mu \tau_{L}^{-1}$ in $T_{m, n}^{(\mu)}$, where $\tau_{L}$ is the longitudinal radiation damping time. Consequently, $\alpha_{R}$ becomes the Robinson damping rate including the effect of radiation damping. Including the effect of radiation damping, Eqs. (B1) and (B3) yield 


$$
\begin{aligned}
& {\left[\left(\Omega-i \alpha_{R}+i \tau_{L}^{-1}\right)^{2}-\omega_{s}^{2}-\frac{i \alpha e \omega_{\mathrm{o}}^{2} I}{2 \pi E} \sum_{n} n Z_{n} F_{n \omega_{\mathrm{o}}}^{2}\right]} \\
& \times\left[\left(\Omega-i \alpha_{R}+2 i \tau_{L}^{-1}\right)^{2}-\left(2 \omega_{s}\right)^{2}-\frac{i \alpha e \omega_{\mathrm{o}}^{2} I}{2 \pi E} \sum_{n} n Z_{n}\left(n \omega_{\mathrm{o}} \sigma_{t}\right)^{2} F_{n \omega_{\mathrm{o}}}^{2}\right] \\
& =\left[\frac{-i \alpha e \omega_{\mathrm{o}}^{2} I}{2 \pi E} \sum_{n} n^{2} Z_{n} F_{n \omega_{\mathrm{o}}}^{2}\right]\left[\frac{-i \alpha e \omega_{\mathrm{o}}^{2} I}{2 \pi E} \sum_{n} Z_{n}\left(n \omega_{\mathrm{o}} \sigma_{t}\right)^{2} F_{n \omega_{\mathrm{o}}}^{2}\right]
\end{aligned}
$$

In a quartic synchrotron potential, Eqs. (B2) and (B3) are approximately obeyed. Above the Landau-damping threshold, we substitute Eq. (A7) for $T_{m, n}^{(\mu)}$ into Eq. (B3) to obtain, including the effects of radiation damping,

$$
\begin{aligned}
& {\left[\left(\Omega-i \alpha_{R}+i \tau_{L}^{-1}\right)^{2}-\left(1.076 \Delta \omega_{s}\right)^{2}-\frac{i \alpha e \omega_{\mathrm{o}}^{2} I}{2 \pi E}(1.076) \sum_{n} n Z_{n} F_{0.93 n \omega_{\mathrm{o}}}^{2}\right]} \\
& \times\left[\left(\Omega-i \alpha_{R}+2 i \tau_{L}^{-1}\right)^{2}-\left(1.164 \cdot 2 \Delta \omega_{s}\right)^{2}-\frac{i \alpha e \omega_{\mathrm{o}}^{2} I}{2 \pi E}(0.982) \sum_{n} n Z_{n}\left(n \omega_{\mathrm{o}} \sigma_{t}\right)^{2} F^{2} \sqrt{2 / 3} n \omega_{\mathrm{o}}\right] \\
& =\left[\frac{-i \alpha e \omega_{\mathrm{o}}^{2} I}{2 \pi E}(1.076) \sum_{n} n^{2} Z_{n} F_{0.93 n \omega_{\mathrm{o}}}^{2}\right]\left[\frac{-i \alpha e \omega_{\mathrm{o}}^{2} I}{2 \pi E}(0.982) \sum_{n} Z_{n}\left(n \omega_{\mathrm{o}} \sigma_{t}\right)^{2} F_{\sqrt{2 / 3}}^{2} n \omega_{\mathrm{o}}\right]
\end{aligned}
$$

Substituting the natural frequency of collective oscillations $\omega_{R}$ for the synchrotron frequency $\omega_{s}$ in Eq. (B1) yields a result which reduces to Eq. (B4) for a quadratic potential, while approximating Eq. (B5) for a quartic potential

$$
\begin{aligned}
& {\left[\left(\Omega-i \alpha_{R}+i \tau_{L}^{-1}\right)^{2}-\omega_{R}^{2}-\frac{i \alpha e \omega_{0}^{2} I}{2 \pi E} \sum_{n} n Z_{n} F_{n \omega_{\mathrm{o}}}^{2}\right]} \\
& \times\left[\left(\Omega-i \alpha_{R}+2 i \tau_{L}^{-1}\right)^{2}-\left(2 \omega_{R}\right)^{2}-\frac{i \alpha e \omega_{\mathrm{o}}^{2} I}{2 \pi E} \sum_{n} n Z_{n}\left(n \omega_{\mathrm{o}} \sigma_{t}\right)^{2} F_{n \omega_{\mathrm{o}}}^{2}\right] \\
& =\left[\frac{-i \alpha e \omega_{\mathrm{o}}^{2} I}{2 \pi E} \sum_{n} n^{2} Z_{n} F_{n \omega_{\mathrm{o}}}^{2}\right]\left[\frac{-i \alpha e \omega_{\mathrm{o}}^{2} I}{2 \pi E} \sum_{n} Z_{n}\left(n \omega_{\mathrm{o}} \sigma_{t}\right)^{2} F_{n \omega_{\mathrm{o}}}^{2}\right] .
\end{aligned}
$$

For $\left|\alpha_{R}\right|, \tau_{L}^{-1} \ll|\Omega|$, Eq. (B6) becomes, for a double-rf system

$$
\begin{aligned}
& \left\{\Omega^{2}-2 i \Omega \alpha_{R}+2 i \Omega \tau_{L}^{-1}-\omega_{R}^{2}+\frac{\alpha e \omega_{g} I}{2 E T_{\mathrm{o}}}\left[R_{1} F_{1}^{2}\left(\sin 2 \phi_{1-}+\sin 2 \phi_{1+}\right)+\nu R_{2} F_{2}^{2}\left(\sin 2 \phi_{2-}+\sin 2 \phi_{2+}\right)\right]\right. \\
& \left.+\frac{i \alpha e \omega_{g} I}{E T_{\mathrm{o}}}\left[R_{1} F_{1}^{2}\left(\cos ^{2} \phi_{1-}-\cos ^{2} \phi_{1+}\right)+\nu R_{2} F_{2}^{2}\left(\cos ^{2} \phi_{2-}-\cos ^{2} \phi_{2+}\right)\right]\right\} \\
& \times\left\{\Omega^{2}-2 i \Omega \alpha_{R}+4 i \Omega \tau_{L}^{-1}-\left(2 \omega_{R}\right)^{2}\right. \\
& +\frac{\alpha e \omega_{g} I}{2 E T_{\mathrm{o}}}\left(\omega_{g} \sigma_{t}\right)^{2}\left[R_{1} F_{1}^{2}\left(\sin 2 \phi_{1-}+\sin 2 \phi_{1+}\right)+\nu^{3} R_{2} F_{2}^{2}\left(\sin 2 \phi_{2-}+\sin 2 \phi_{2+}\right)\right] \\
& \left.+\frac{i \alpha e \omega_{g} I}{E T_{\mathrm{o}}}\left(\omega_{g} \sigma_{t}\right)^{2}\left[R_{1} F_{1}^{2}\left(\cos ^{2} \phi_{1-}-\cos ^{2} \phi_{1+}\right)+\nu^{3} R_{2} F_{2}^{2}\left(\cos ^{2} \phi_{2-}-\cos ^{2} \phi_{2+}\right)\right]\right\} \\
& =\left(\omega_{g} \sigma_{t}\right)^{2}\left\{\frac{\alpha e \omega_{g} I}{2 E T_{\mathrm{o}}}\left[R_{1} F_{1}^{2}\left(\sin 2 \phi_{1-}-\sin 2 \phi_{1+}\right)+\nu^{2} R_{2} F_{2}^{2}\left(\sin 2 \phi_{2-}-\sin 2 \phi_{2+}\right)\right]\right. \\
& \left.\quad+\frac{i \alpha e \omega_{g} I}{E T_{\mathrm{o}}}\left[R_{1} F_{1}^{2}\left(\cos ^{2} \phi_{1-}+\cos ^{2} \phi_{1+}\right)+\nu^{2} R_{2} F_{2}^{2}\left(\cos ^{2} \phi_{2-}+\cos ^{2} \phi_{2+}\right)\right]\right\}^{2}
\end{aligned}
$$

Defining the real quantities 


$$
\begin{aligned}
\tilde{A} & \equiv \frac{\alpha e \omega_{g} I}{2 E T_{\mathrm{o}}}\left[R_{1} F_{1}^{2}\left(\sin 2 \phi_{1-}+\sin 2 \phi_{1+}\right)+\nu R_{2} F_{2}^{2}\left(\sin 2 \phi_{2-}+\sin 2 \phi_{2+}\right)\right], \\
\tilde{B} & \equiv \frac{\alpha e \omega_{g} I}{2 E T_{\mathrm{o}}}\left(\omega_{g} \sigma_{t}\right)^{2}\left[R_{1} F_{1}^{2}\left(\sin 2 \phi_{1-}+\sin 2 \phi_{1+}\right)+\nu^{3} R_{2} F_{2}^{2}\left(\sin 2 \phi_{2-}+\sin 2 \phi_{2+}\right)\right], \\
\tilde{D} & \equiv\left(\omega_{g} \sigma_{t}\right) \frac{\alpha e \omega_{g} I}{2 E T_{\mathrm{o}}}\left[R_{1} F_{1}^{2}\left(\sin 2 \phi_{1-}-\sin 2 \phi_{1+}\right)+\nu^{2} R_{2} F_{2}^{2}\left(\sin 2 \phi_{2-}-\sin 2 \phi_{2+}\right)\right], \\
\tilde{a} & \equiv \frac{\alpha e \omega_{g} I}{E T_{\mathrm{o}}}\left[R_{1} F_{1}^{2}\left(\cos ^{2} \phi_{1-}-\cos ^{2} \phi_{1+}\right)+\nu R_{2} F_{2}^{2}\left(\cos ^{2} \phi_{2-}-\cos ^{2} \phi_{2+}\right)\right]+2 \Omega \tau_{L}^{-1}, \\
\tilde{b} & \equiv \frac{\alpha e \omega_{g} I}{E T_{\mathrm{o}}}\left(\omega_{g} \sigma_{t}\right)^{2}\left[R_{1} F_{1}^{2}\left(\cos ^{2} \phi_{1-}-\cos ^{2} \phi_{1+}\right)+\nu^{3} R_{2} F_{2}^{2}\left(\cos ^{2} \phi_{2-}-\cos ^{2} \phi_{2+}\right)\right]+4 \Omega \tau_{L}^{-1}, \\
\tilde{d} & \equiv\left(\omega_{g} \sigma_{t}\right) \frac{\alpha e \omega_{g} I}{E T_{\mathrm{o}}}\left[R_{1} F_{1}^{2}\left(\cos ^{2} \phi_{1-}+\cos ^{2} \phi_{1+}\right)+\nu^{2} R_{2} F_{2}^{2}\left(\cos ^{2} \phi_{2-}+\cos ^{2} \phi_{2+}\right)\right],
\end{aligned}
$$

Eq. (B7) becomes

$$
\left[\Omega^{2}-\omega_{R}^{2}+\tilde{A}-2 i \Omega \alpha_{R}+i \tilde{a}\right]\left[\Omega^{2}-\left(2 \omega_{R}\right)^{2}+\tilde{B}-2 i \Omega \alpha_{R}+i \tilde{b}\right]=[\tilde{D}+i \tilde{d}]^{2} .
$$

The real part of Eq. (B9) yields

$$
\left[\Omega^{2}-\omega_{R}^{2}+\tilde{A}\right]\left[\Omega^{2}-\left(2 \omega_{R}\right)^{2}+\tilde{B}\right]=\tilde{D}^{2}-\tilde{d}^{2}+\left(\tilde{a}-2 \Omega \alpha_{R}\right)\left(\tilde{b}-2 \Omega \alpha_{R}\right),
$$

while its imaginary part yields the Robinson damping rate (including radiation damping)

$$
\alpha_{R}=\frac{\tilde{a}\left[\Omega^{2}-\left(2 \omega_{R}\right)^{2}+\tilde{B}\right]+\tilde{b}\left[\Omega^{2}-\omega_{R}^{2}+\tilde{A}\right]-2 \tilde{D} \tilde{d}}{2 \Omega\left[2 \Omega^{2}-5 \omega_{R}^{2}+\tilde{A}+\tilde{B}\right]} .
$$

A zero-frequency instability threshold is predicted when Eq. (B10) is obeyed for $\Omega^{2} \rightarrow 0$, in which case $\phi_{1 \pm} \rightarrow \phi_{1}$ and $\phi_{2 \pm} \rightarrow \phi_{2}$ while $\tilde{D}, \tilde{a}, \tilde{b} \rightarrow 0$. This yields

$$
0=\left.\left[\left(\omega_{R}^{2}-\tilde{A}\right)\left(4 \omega_{R}^{2}-\tilde{B}\right)+\tilde{d}^{2}\right]\right|_{\substack{\phi_{1+=\phi_{1}-=\phi_{1}} \\ \phi_{2+}=\phi_{2}-=\phi_{2}}} .
$$

This threshold cannot be reached without first crossing the threshold of the equilibrium phase instability $\left(\omega_{R}^{2}-\tilde{A}=0\right)$ or zero-frequency quadrupole Robinson instability $\left(4 \omega_{R}^{2}-\tilde{B}=0\right)$. These thresholds occur when there is no restoring force for slow perturbations of the bunch position or length, in which case fast instabilities may result that involve many synchrotron modes [15]. Because of the contribution of many synchrotron modes to fast instabilities, the coupled dipole-quadrupole model (which includes only the dipole and quadrupole modes) may no longer be applicable when the equilibrium phase instability or zero-frequency quadrupole instability thresholds are reached.

Application of the quadratic formula to Eq. (B10) yields

$$
\Omega^{2}=\frac{5 \omega_{R}^{2}-\tilde{A}-\tilde{B}}{2} \pm\left[\frac{\left(3 \omega_{R}^{2}+\tilde{A}-\tilde{B}\right)^{2}}{4}+\tilde{D}^{2}-\tilde{d}^{2}+\left(\tilde{a}-2 \Omega \alpha_{R}\right)\left(\tilde{b}-2 \Omega \alpha_{R}\right)\right]^{1 / 2} .
$$

When the minus sign is taken for \pm , we refer to the solution as the "coupled-dipole" mode, while the plus sign gives the "coupled-quadrupole" mode. In the absence of coupling $(\tilde{D}=\tilde{d}=0)$, Eqs. (B11)-(B13) reproduce the uncoupled dipole and quadrupole mode results of Eqs. (13)-(16). The coupling term $\tilde{D}$ resulting from the imaginary part of the impedance pulls the coupled-dipole and coupled-quadrupole mode frequencies apart, while the coupling term $\tilde{d}$ resulting from the real part of the impedance pushes the coupled-dipole and coupled-quadrupole mode frequencies together. Consequently, the mode-coupling behavior with a passive Landau cavity (where the impedance is mostly imaginary) may differ from that of an active Landau cavity (where the effective impedance, including feedback, is mostly real).

When $\tilde{d}$ is sufficiently large, the argument of the square root goes to zero in Eq. (B13); for larger values of $\tilde{d}$ a real value of $\Omega^{2}$ is inconsistent with Eq. (B13). In this case, slowly growing or damping Robinson oscillations do not exist because of strong mode coupling, indicating a fast mode-coupling instability [15,22]. At the fast mode-coupling threshold, the coupled-dipole and coupled-quadrupole frequencies are equal. When a fast instability occurs, many synchrotron modes may contribute to the unstable motion [15]. 
[1] A. Hofmann and S. Myers, in Proceedings of the 11th International Conference on High Energy Accelerators, Geneva (Birkhäuser Verlag, Basel, 1980), p. 610.

[2] E. Weihreter, A. Gaupp, H. G. Hoberg, W.-D. Klotz, P. Kuske, H. Lehr, R. Maier, and G. Mülhaupt, IEEE Trans. Nucl. Sci. 32, 2317 (1985).

[3] Y. Miyahara, S. Asaoka, A. Mikuni, and K. Soda, Nucl. Instrum. Methods Phys. Res., Sect. A 260, 518 (1987).

[4] S. Krinsky, J. Bittner, A. M. Fauchet, E. D. Johnson, J. Keane, J. Murphy, R. J. Nawrocky, J. Rogers, O. V. Singh, and L.H. Yu, in The Physics of Particle Accelerators, edited by M. Month and M. Dienes, AIP Conf. Proc. No. 249 (AIP, New York, 1992), p. 762.

[5] R. A. Bosch and C. S. Hsue, Part. Accel. 42, 81 (1993).

[6] K. J. Kleman, in Proceedings of the 1995 Particle Accelerator Conference, Dallas (IEEE, Piscataway, NJ, 1996), p. 1785.

[7] M. Migliorati, L. Palumbo, and M. Zobov, Nucl. Instrum. Methods Phys. Res., Sect. A 354, 215 (1995).

[8] M. Georgsson, Å. Andersson, and M. Eriksson, Nucl. Instrum. Methods Phys. Res., Sect. A 416, 465 (1998).

[9] J. M. Byrd, S. De Santis, M. Georgsson, G. Stover, J. D. Fox, and D. Teytelman, Nucl. Instrum. Methods Phys. Res., Sect. A 455, 271 (2000).

[10] K. W. Robinson, Cambridge Electron Accelerator Report No. CEA-11, 1956.

[11] R. A. Bosch, in Proceedings of the 1997 Particle Accelerator Conference, Vancouver, Canada (IEEE, Piscataway, NJ, 1998), p. 862.

[12] Tai-Sen F. Wang, Part. Accel. 34, 105 (1990).

[13] Tai-Sen F. Wang, in Proceedings of the 1993 Particle Accelerator Conference, Washington, DC (IEEE, Piscataway, NJ, 1993), p. 3500.

[14] P. Marchand, Part. Accel. 36, 205 (1992).

[15] S. Krinsky and J. M. Wang, Part. Accel. 17, 109 (1985).
[16] N. Towne and J. M. Wang, Phys. Rev. E 57, 3461 (1998).

[17] R. A. Bosch, Synchrotron Radiation Center Technical Note No. SRC-137, 1994.

[18] J. J. Bisognano, R. A. Bosch, D. E. Eisert, M. A. Green, K. J. Kleman, and W.S. Trzeciak, in Proceedings of the 2001 Particle Accelerator Conference, Chicago (to be published).

[19] M. Sands, Institut National de Physique Nucleaire et de Physique des Particules, Rapport technique 2-76, 3-76, and 4-76, 1976.

[20] P. B. Wilson, in Physics of High Energy Particle Accelerators, edited by R. A. Carrigan, F. R. Huson, and M. Month, AIP Conf. Proc. No. 87 (AIP, New York, 1982), p. 450.

[21] J. Haïssinski, Nuovo Cimento Soc. Ital Fis. 18B, 72 (1973).

[22] J. M. Wang, in Physics of Particle Accelerators, edited by M. Month and M. Deines, AIP Conf. Proc. No. 153 (AIP, New York, 1987), p. 697.

[23] J.M. Wang, Brookhaven National Laboratory Report No. BNL 51302, 1980.

[24] M.S. Zisman, S. Chattopadhyay, and J. J. Bisognano, Lawrence Berkeley Laboratory Report No. LBL-21270, 1986.

[25] S. Haykin, Communication Systems (Wiley, New York, 1994).

[26] S.P. Jachim and E. F. Natter, in Proceedings of the 1989 IEEE Particle Accelerator Conference, Chicago (IEEE, Piscataway, NJ, 1989), p. 1870.

[27] P.B. Wilson, K. L.F. Bane, and K. Satoh, IEEE Trans. Nucl. Sci. 28, 2525 (1981).

[28] R. H. Siemann, IEEE Trans. Nucl. Sci. 30, 2373 (1983).

[29] R. A. Bosch, K. J. Kleman, and J. J. Bisognano, in Proceedings of the 2001 Particle Accelerator Conference, Chicago (Ref. [18]).

[30] D. R. Nicholson, Introduction to Plasma Theory (Wiley, New York, 1983). 